\title{
A Convergence Theorem for Lattice Feynman Integrals with Massless Propagators
}

\author{
Thomas Reisz ${ }^{\star}$ \\ Deutsches Elektronen-Synchrotron DESY, D-2000 Hamburg, Federal Republic of Germany
}

\begin{abstract}
It is shown that for non-vanishing lattice spacing, conventional infrared power counting conditions are sufficient for convergence of lattice Feynman integrals with zero-mass propagators. If these conditions are supplemented by ultraviolet convergence conditions, the continuum limit of such a diagram exists and is universal.
\end{abstract}

\section{Introduction}

In a recent paper [1] we have proposed a convergence theorem, which states existence of the continuum limit for a wide class of Feynman integrals with a lattice cutoff if certain ultraviolet (UV) power counting conditions are satisfied. What is counted are lattice divergence degrees in Zimmermann subspaces, i.e. in affine subspaces of the integration momenta. To avoid infrared (IR) singularities, we had assumed all propagators to be massive. In the present article we extend the considerations to integrals containing zero-mass propagators. While the lattice provides a UV-cutoff, IR-singularities are expected to be quite the same as for continuum diagrams. As will be shown, IR-power counting conditions similar as for continuum diagrams [2-5] are sufficient to guarantee the convergence of lattice Feynman integrals, at least for non-vanishing lattice spacing. If these conditions are supplemented by the UV-power counting conditions of [1], the continuum limit of the Feynman integral exists and coincides with the formal limit, i.e. it is given by the integral resulting from the $a \rightarrow 0$-limit in the integrand.

This article is organized as follows. At first, in Sect. 2, the notion of an IR-degree is introduced in a form which is similar to the definition of a UV-degree in [1]. The power counting theorem for Feynman integrals with zero-mass propagators is formulated in Sect. 3. As in the massive case, the denominator of a Feynman integrand can easily be treated, whereas the numerator must be estimated in such a way that UV- as well as IR-power counting conditions are taken into account

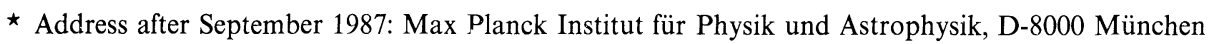
40, Federal Republic of Germany 
(Sect. 5), and such that the corresponding estimates have a well defined cutoff behavior. This behavior can be determined using the auxiliary theorem stated in Sect. 4. Applying the auxiliary theorem and using the estimate of the numerator, the proof of the power counting theorem is given in Sect. 6. Finally, the last two sections are devoted to the proof of the auxiliary theorem.

\section{IR-Degrees on the Lattice}

Throughout this paper we will use the notations, definitions and statements of [1], especially the function classes $\mathscr{C}_{m}, \mathscr{C}$ and $\mathscr{C}_{m}^{c}, \mathscr{C}^{c}$ and $\mathscr{F}$. We shall use multi-indices to simplify the notation. Set $\mathbf{N}_{0}=\mathbf{N} \cup\{0\}=\{0,1,2, \ldots\}$. For $b \in \mathbf{N}_{0}^{n}, u \in \mathbf{R}^{n}$ define

$$
b !=b_{1} ! \cdots b_{n} !, \quad u^{b}=u_{1}^{b_{1}} \cdots u_{n}^{b_{n}}, \quad|b|=\sum_{i=1}^{n} b_{i} .
$$

We now define an IR-degree for functions in $\mathscr{C}$, depending on variables $u$ ("internal" momenta), $v, q$ ("external" momenta) and the lattice spacing $a$.

Definition 2.1. 1. Let $m \in \mathbf{Z}$ and $V(u, v, q ; a) \in \mathscr{C}_{m}$ of the form

$$
V(u, v, q ; a)=\frac{1}{a^{m}} F(u a, v a, q a) .
$$

For given $q$ let $s_{u}$ be the largest non-negative integer such that

$$
\left(\frac{\partial^{c}}{\partial u^{c}} F(u a, v a, q a)\right)_{u=0} \equiv 0 \text { in } v \text { and } a>0, \text { for all } c \in \mathbf{N}_{0}^{s}, \quad|c|<s_{u} \text {. }
$$

Then the IR-degree of $V$ with respect to $u$ is defined by

$$
\underline{\operatorname{degr}}_{\hat{u} \mid v} V=s_{u} .
$$

2. Let $V \in \mathscr{C}, V=\sum_{i \in I} V_{i}, V_{i} \in \mathscr{C}_{m_{i}}$ for some $m_{i} \in \mathbf{Z}, m_{i} \neq m_{k}$ for $i \neq k$. Then we define

$$
\underline{\operatorname{degr}}_{\hat{u} \mid v} V=\min _{i \in I} \underline{\operatorname{degr}}_{\hat{u} \mid v} V_{i} .
$$

An equivalent definition is the following. For $V \in \mathscr{C}, s_{u}=\underline{\operatorname{degr}}_{\hat{\imath} \mid v} V$ if and only if

$$
V(\lambda u, v, q ; a)=B(u, v, q ; a) \lambda^{s_{u}}+O\left(\lambda^{s_{u}+1}\right), \quad \lambda \rightarrow 0,
$$

where $B(u, v, q ; a) \neq 0$ in $u, v, a$, for fixed $q\left(B\right.$ is a polynomial in $u$ and $C^{\infty}$ in $\left.v\right)$.

It is important to note that this IR-degree may depend on the external momenta $q$. Following common use, we write all momentum variables which are not fixed as subscript in degr, e.g. $u, v$ in (2-3). If $\partial^{c} F(u a, v a, q a) /\left.\partial u^{c}\right|_{u=0} \equiv 0$ in $v$ and $a$ for all $c \in \mathbf{N}_{0}^{s}$, we set $\overline{\operatorname{degr}}_{\hat{u} \mid v} V=+\infty$. If $V(u, v, q ; a) \neq 0$ in $u, v, a$ and independent of $u$, then $\operatorname{degr}_{\hat{u} \mid v} V=0$.

From the definition of an IR-degree, we easily get

Lemma 2.1. Let $V_{1}, \ldots, V_{p} \in \mathscr{C}$. Then

$$
\text { 1. } \underline{\operatorname{degr}}_{\hat{u} \mid v} \sum_{i=1}^{p} V_{i} \geqq \min _{i=1, \ldots, p} \underline{\operatorname{degr}} \hat{u}|v|
$$




$$
\begin{aligned}
& \text { 2. } \underline{\operatorname{degr}}_{\hat{u} \mid v} \prod_{i=1}^{p} V_{i} \geqq \sum_{i=1}^{p} \underline{\operatorname{degr}}_{\hat{u} \mid v} V_{i}, \\
& \text { 3. } \underline{\operatorname{degr}}_{\hat{u} \mid v} \frac{\partial^{l}}{\partial u^{l}} V \geqq \underline{\operatorname{degr}_{\hat{u} \mid v} V-|l|,} \\
& \text { 4. } \underline{\operatorname{degr}}_{\hat{u} \mid v} \frac{\partial^{l}}{\partial v^{l}} V \geqq \underline{\operatorname{degr}}_{\hat{u} \mid v} V .
\end{aligned}
$$

Next, we consider functions in the classes $\mathscr{C}_{m}^{c}$ and $\mathscr{C}^{c}$, i.e. functions in $\mathscr{C}_{m}$ and $\mathscr{C}$ whose continuum limits exist. Every $V \in \mathscr{C}_{m}^{c}$ has an expansion for small lattice spacing $a$ of the form

$$
V(u, v, q ; a)=\frac{1}{a^{m}} F(u a, v a, q a)=P(u, v, q)+R(u, v, q ; a),
$$

where the continuum limit $P$ of $V$ is a homogeneous polynomial in $u, v, q$ and $R$ vanishes for $a=0$. In general,

$$
\underline{\operatorname{degr}}_{u \mid v} P \geqq \underline{\operatorname{degr}}_{\hat{u} \mid v} V,
$$

where the IR-degree of a polynomial is defined in Appendix A. In particular, with respect to all momentum variables $u, v$ and $q$

$$
\underline{\operatorname{degr}}_{u v q} P=\underline{\operatorname{degr}_{\widehat{u v q}} V} \text { if } P(u, v, q) \neq 0 .
$$

In Sect. 5 we will state a general estimate on the remainder $R$ which respects the IR- and UV-properties of the function $V$ and allows to determine the cutoff behavior of Feynman integrals having $R$ as the numerator of the integrand, by application of an auxiliary theorem stated in Sect. 4 , which is a generalization of the auxiliary power counting theorem in [1] to diagrams with massless propagators.

The integrand of a Feynman integral on the lattice belongs to the function class $\mathscr{F}[1]$. For $F \in \mathscr{F}$ an IR-degree is defined as follows.

Definition 2.2. Let $F \in \mathscr{F}$,

$$
F(u, v, q ; \mu, a)=\frac{V(u, v, q ; \mu, a)}{C(u, v, q ; \mu, a)} .
$$

Then the IR-degree of $F$ with respect to $u$ is defined by

$$
\underline{\operatorname{degr}} \hat{\hat{u} \mid v}, \underline{\operatorname{degr}_{\hat{u} \mid v}} V-\underline{\operatorname{degr}}_{\hat{u} \mid v} C .
$$

Recall that the denominator in (2-11) is of the form

$$
C=\prod_{i=1}^{n}\left(\frac{\eta_{i}\left(l_{i} a\right)}{a^{2}}+\mu_{i}^{2}\right), \quad \mu_{i}^{2} \geqq 0,
$$

where the four-vectors $l_{i} \neq 0$ are given by

$$
l_{i}(u, v, q)=\sum_{k=1}^{d} b_{i k} v_{k}+\sum_{k=1}^{r} c_{i k} u_{k}+\sum_{k=1}^{w} d_{i k} q_{k} .
$$


The IR-degree of the denominator is already determined by the IR-degree of its continuum limit:

$$
\begin{aligned}
& \underline{\operatorname{degr}}_{\hat{u} \mid v}\left(\frac{\eta_{i}\left(l_{i} a\right)}{a^{2}}+\mu_{i}^{2}\right)=\underline{\operatorname{degr}}_{\hat{u} \mid v}\left(l_{i}^{2}+\mu_{i}^{2}\right) \\
& =\left\{\begin{array}{llll}
0 & \text { if } \mu_{i}^{2}>0 & \text { or } \quad\left(b_{i 1}, \ldots, b_{i d}\right) \neq 0 \text { or } \sum_{k=1}^{w} d_{i k} q_{k} \neq 0 \\
2 & \text { if } \mu_{i}^{2}=0 & \text { and } \quad\left(b_{i 1}, \ldots, b_{i d}\right)=0 \text { and } \sum_{k=1}^{w} d_{i k} q_{k}=0 .
\end{array}\right.
\end{aligned}
$$

Note that

$$
\underline{\operatorname{degr}}_{\hat{u} \mid v} \prod_{i=1}^{n}\left(\frac{\eta_{i}\left(l_{i} a\right)}{a^{2}}+\mu_{i}^{2}\right)=\sum_{i=1}^{n} \underline{\operatorname{degr}_{\hat{u} \mid v}}\left(\frac{\eta_{i}\left(l_{i} a\right)}{a^{2}}+\mu_{i}^{2}\right),
$$

and for every $F \in \mathscr{F}$,

$$
\underline{\operatorname{degr}}_{u \mid v} \lim _{a \rightarrow 0} F(u, v, q ; \mu, a) \geqq \underline{\operatorname{degr}}_{\hat{u} \mid v} F(u, v, q ; \mu, a) .
$$

Finally, as a corollary of Lemma 2.1, we state

Lemma 2.2. Let $F, F_{1}, \ldots, F_{p} \in \mathscr{F}$. Then

$$
\begin{aligned}
& \text { 1. } \underline{\operatorname{degr}}_{\hat{u} \mid v} \sum_{i=1}^{p} F_{i} \geqq \min _{i=1, \ldots, p} \operatorname{degr}_{\hat{u} \mid v} F_{i}, \\
& \text { 2. } \underline{\operatorname{degr}}_{\hat{u} \mid v} \prod_{i=1}^{p} F_{i} \geqq \sum_{i=1}^{p} \underline{\operatorname{degr}_{\hat{u} \mid v} F_{i},} \\
& \text { 3. } \underline{\operatorname{degr}}_{\hat{u} \mid v} \frac{\partial^{l}}{\partial u^{l}} F \geqq \underline{\operatorname{degr}_{\hat{u} \mid v} F-|l|,} \\
& \text { 4. } \underline{\operatorname{degr}}_{\hat{u} \mid v} \frac{\partial^{l}}{\partial v^{l}} F \geqq \underline{\operatorname{degr}}_{\hat{u} \mid v} F .
\end{aligned}
$$

\section{The Power Counting Theorem for Feynman Integrals with Massless Propagators}

We consider

$$
\hat{I}(q ; \mu, a)=\int_{-\pi / a}^{\pi / a} d^{4} k_{1} \cdots d^{4} k_{m} F(k, q ; \mu, a)
$$

where

$$
\begin{aligned}
& F(k, q ; \mu, a)=V(k, q ; \mu, a) / C(k, q ; \mu, a) \in \mathscr{F}, \\
& C(k, q ; \mu, a)=\prod_{i=1}^{n}\left[\frac{\eta_{i}\left(l_{i}(k, q) a\right)}{a^{2}}+\mu_{i}^{2}\right], \quad \mu_{i}^{2} \geqq 0
\end{aligned}
$$

(vanishing masses are allowed). Furthermore, let $\mathscr{L}$ be a natural set of fourmomenta containing $l_{1}, \ldots, l_{n}[1]$. At first, we repeat the definition of UV-divergence degrees [1] and then define IR-divergence degrees.

1. Let

$$
u_{1}=l_{i_{1}}, \ldots, u_{d}=l_{i_{d}}, \quad v_{1}=l_{j_{1}}, \ldots, v_{m-d}=l_{j_{m-d}}
$$


be an arbitrary basis of $\mathscr{L}$ with respect to $k^{1}, 1 \leqq d \leqq m$. By fixing $v_{1}, \ldots, v_{m-d}$, one defines a class $H$ of affine subspaces of the space of integration momenta $k$. $(u)=\left(u_{1}, \ldots, u_{d}\right)$ is called the parametrization of $H$, and $(v)=\left(v_{1}, \ldots, v_{d}\right)$ are the complementary parameters of $H$. As in [1], we define for $F(k, q ; \mu, a) \in \mathscr{F}$,

$$
\overline{\operatorname{degr}}_{\hat{H}} \hat{I}(q ; \mu, a) \equiv 4 d+\overline{\operatorname{degr}}_{\hat{u}} F(k(u, v, q), q ; \mu, a) .
$$

The set of all such $H$, for all bases (3-2), is denoted by $\mathscr{H}^{\mathrm{Uv}}$ (this is the set $\mathscr{H}$ of Zimmermann subspaces of [1]. Here we write $\mathscr{H}^{\mathrm{UV}}$ to distinguish this set of subspaces from the set $\mathscr{H}^{\mathrm{IR}}$ defined below).

2. Let $\mathscr{M}=\left\{l_{i} \mid \mu_{i}=0 ; i=1, \ldots, n\right\} \subseteq \mathscr{L}$. For every basis (3-2) such that

$$
l_{i_{1}}, \ldots, l_{i_{d}} \in \mathscr{M}
$$

and $d \geqq 1$, we define a subspace $H$ as above. The set of all these $H$ is denoted by $\mathscr{H}^{\mathrm{IR}}$. Obviously, $\mathscr{H}^{\mathrm{IR}} \subseteq \mathscr{H}^{\mathrm{UV}}$. For $H \in \mathscr{H}^{\mathrm{IR}}$ we define the IR-divergence degree

$$
\underline{\operatorname{degr}}_{\hat{H}} \hat{I}(q ; \mu, a) \equiv 4 d+\underline{\operatorname{degr}}_{\hat{u} \mid v} F(k(u, v, q), q ; \mu, a) .
$$

We now state the power counting theorem which applies to lattice Feynman integrals with massless propagators.

Theorem 1. Power Counting Theorem. Consider the integral

$$
\hat{I}(q ; \mu, a)=\int_{-\pi / a}^{\pi / a} d^{4} k_{1} \cdots d^{4} k_{m} F(k, q ; \mu, a),
$$

and suppose the integrand is of the form

$$
F(k, q ; \mu, a)=\frac{V(k, q ; \mu, a)}{C(k, q ; \mu, a)} \in \mathscr{F},
$$

where $V \in \mathscr{C}^{c}$ is $(2 \pi / a)$-periodic in every component of $k$, and

$$
C(k, q ; \mu, a)=\prod_{i=1}^{n}\left[\frac{\eta_{i}\left(l_{i}(k, q) a\right)}{a^{2}}+\mu_{i}^{2}\right], \quad \mu_{i}^{2} \geqq 0 .
$$

Suppose, furthermore, the line momenta $l_{i}$ are contained in a natural set $\mathscr{L}$ of momenta and assume that for every $H \in \mathscr{H}^{\mathrm{IR}}$

$$
\operatorname{degr}_{\hat{H}} \hat{I}(q ; \mu, a)>0 .
$$

Then the integral (3-1) is absolutely convergent for every $a>0$. If, in addition, for every $H \in \mathscr{H}^{\mathrm{UV}}$ we have

$$
\overline{\operatorname{degr}}_{\hat{H}} \hat{I}(q ; \mu, a)<0,
$$

the continuum limit of $\hat{I}(q ; \mu, a)$ exists absolutely and is given by

$$
\lim _{a \rightarrow 0} \hat{I}(q ; \mu, a)=\int_{-\infty}^{\infty} d^{4} k_{1} \cdots d^{4} k_{m} \frac{P(k, q, \mu)}{E(k, q, \mu)},
$$

\footnotetext{
${ }^{1}$ cp. [1] or Sect. 4 below. $u_{1}, \ldots, u_{d}, v_{1}, \ldots, v_{m-d} \in \mathscr{L}$, and the Jacobian satisfies $\operatorname{det}[\partial(u, v) / \partial k] \neq 0$.
} There is at least one basis of $\mathscr{L}$ with respect to $k$ 
where

$$
\begin{aligned}
& P(k, q, \mu)=\lim _{a \rightarrow 0} V(k, q ; \mu, a), \\
& E(k, q, \mu)=\lim _{a \rightarrow 0} C(k, q ; \mu, a) .
\end{aligned}
$$

Note that vanishing masses are allowed, and that convergence is stated for given (fixed) external momenta $q$. Furthermore, the integrand is always assumed to be periodic. As in the massive case [1], if $P \neq 0$, the set $\mathscr{L}^{\prime}=\left\{l_{1}, \ldots, l_{n}\right\}$ contains a basis of $\mathscr{L}$ with respect to $k$ (otherwise (3-8) would be UV-divergent). Hence, in this case it is sufficient to consider $\mathscr{L}^{\prime}$ instead of $\mathscr{L}$. Note that

$$
\overline{\operatorname{degr}}_{u} \lim _{a \rightarrow 0} F(u, v, q ; \mu, a) \leqq \overline{\operatorname{degr}}_{\hat{u}} F(u, v, q ; \mu, a)
$$

and

$$
\underline{\operatorname{degr}}_{u \mid v} \lim _{a \rightarrow 0} F(u, v, q ; \mu, a) \geqq \underline{\operatorname{degr}}_{\hat{u} \mid v} F(u, v, q ; \mu, a) .
$$

Hence, by (3-6), (3-7) and the power counting theorem of Lowenstein and Zimmermann $[2,3]^{2},(3-8)$ is absolutely convergent.

The idea of proof is quite similar to that of the power counting theorem for Feynman integrals with massive propagators [1]. Again, it will be sufficient to consider

$$
\hat{I}(q ; \mu, a)=\int_{-\pi / a}^{\pi / a} d^{4} k_{1} \cdots d^{4} k_{m} \frac{V(k, q ; a)}{\prod_{i=1}^{n}\left(\frac{\eta_{i}\left(l_{i} a\right)}{a^{2}}+\mu_{i}^{2}\right)},
$$

where $V \in \mathscr{C}_{m_{0}}^{c}$ for some $m_{0} \in \mathbf{Z}$. Without loss of generality we also assume $\mathscr{L}$ to be of the form $\left\{l_{1}, \ldots, l_{N}\right\}$ for some $N \geqq n$, and that $k_{1}, \ldots, k_{m}$ belong to $\mathscr{L}$.

In the first step of the proof, the integration domain of (3-12) is partitioned in a way depending on the configuration of the line momenta $l_{i}$. It is distinguished between $l_{i}$ in neighborhoods of the poles of propagators and outside of them. A propagator can be estimated by its continuum limit or some power of the lattice spacing $a$, respectively. Again, the numerator causes some technical problems, and we need an estimation which respects UV- as well as IR-degrees. In the next section we state an auxiliary theorem which describes the cutoff dependence of generalized continuum Feynman integrals with zero-mass propagators. Then, in Sect. 5 it is shown that the numerator of (3-11) admits an estimate such that this auxiliary theorem applies to the integrals resulting from the partition of (3-11) explained above.

\section{A Power Counting Theorem for Generalized Continuum Feynman Integrals with Zero-Mass Propagators}

To formulate the auxiliary theorem, we will use the notations of the auxiliary power counting theorem of [1]. For completeness, they will be repeated here.

${ }^{2}$ Or by the auxiliary theorem below 
Let $k=\left(k_{1}, \ldots, k_{m}\right)$ (loop momenta) and $q=\left(q_{1}, \ldots, q_{M}\right)$ (external momenta), $k_{i}, q_{j} \in \mathbf{R}^{4}$, and let $L$ denote the space of linear mappings $l: \mathbf{R}^{4 m} \times \mathbf{R}^{4 M} \rightarrow \mathbf{R}^{4}$ of the form

$$
\begin{aligned}
l(k, q) & =K(k)+Q(q), \\
K(k) & =\sum_{i=1}^{m} a_{i} k_{i} ; \quad a_{i} \in \mathbf{R}, \quad i=1, \ldots, m, \\
Q(q) & =\sum_{j=1}^{M} b_{j} q_{j} ; \quad b_{j} \in \mathbf{R}, \quad j=1, \ldots, M .
\end{aligned}
$$

Elements $l_{1}, \ldots, l_{s}$ are called linearly independent with respect to $k$ if their homogeneous parts in $k$ are linearly independent. $\left\{l_{1}, \ldots, l_{s}\right\} \subseteq \mathscr{M} \subset L$ is called a basis of $\mathscr{M}$ with respect to $k$ if every $l \in \mathscr{M}$ has a unique representation

$$
l(k, q)=\sum_{i=1}^{s} c_{i} l_{i}(k, q)+Q(q),
$$

where $c_{i} \in \mathbf{R} ; i=1, \ldots, s$ and $Q$ is linear. We define $\operatorname{rank}_{k} \mathscr{M}=s$.

Let $\mathscr{L} \subset L$ be a finite subset

where

$$
\mathscr{L}=\left\{l_{i}(k, q)=\sum_{j=1}^{m} C_{i j} k_{j}+Q_{i}(q) \mid i=1, \ldots, N\right\}
$$

$$
\begin{aligned}
\operatorname{rank}\left(C_{i j}\right) & =m, \\
\left(C_{i 1}, \ldots, C_{i m}\right) & \neq 0 \text { for all } i=1, \ldots, N, \\
l_{i}^{2} & \neq l_{j}^{2} \text { if } i \neq j,
\end{aligned}
$$

so that $\operatorname{rank}_{k} \mathscr{L}=m$. Let $\mathscr{N} \subseteq \mathscr{L}$. We consider the behavior of the integral

for large $\lambda$. Here

$$
\bar{I}_{\lambda}(q, \mu)=\int^{\lambda, \mathscr{L}} d^{4} k_{1} \cdots d^{4} k_{m} \frac{Z(\lambda, k, q)}{E(k, q, \mu)}
$$

$$
E(k, q, \mu)=\prod_{\mathscr{N}}\left(l_{i}^{2}(k, q)+\mu_{i}^{2}\right)^{n_{2}}, \quad \mu_{i}^{2} \geqq 0, \quad n_{i} \in \mathbf{N}=\{1,2, \ldots\},
$$

and $\prod_{\mathscr{N}}$ means the product over all $l_{i} \in \mathscr{N}$. Note that vanishing masses are allowed. The integration runs over all $k \in \mathbf{R}^{4 m}$ constrained by $l_{i}^{2}(k, q) \leqq \lambda^{2}, i=1, \ldots, N$. The numerator is of the form

$$
Z(\lambda, k, q)=\min _{i \in I}\left(\min _{j \in J_{i}}\left|M_{i j}(k, q)\right| \cdot \min _{l \in K_{i}} \lambda^{-p_{l} \mid}\left|C_{i l}(k, q)\right|\right),
$$

where $I, J_{i}, K_{i}$ are finite sets, $p_{i l} \in \mathbf{N}_{0}=\{0,1,2, \ldots\}$, and $M_{i j}, C_{i l}$ are polynomials. $I$ subscripts the set of all $\mathscr{S} \subseteq \mathscr{S}_{0}$ (including $\mathscr{S}=\emptyset$ ), where

$$
\mathscr{S}_{0}=\left\{l_{i} \in \mathcal{N} \mid \mu_{i}=0\right\} \text {. }
$$

In the following, a function which is of the form (4-8) will be called a nominator function. 
Next, we define the sets $\mathscr{H}^{\mathrm{UV}}$ and $\mathscr{H}^{\mathrm{IR}}$ as in Sect. 3, but instead of (3-4) we now let $l_{i_{1}}, \ldots, l_{i_{d}} \in \mathscr{S}_{0}$ for defining $\mathscr{H}^{\mathrm{IR}}$, and $\mathscr{L}$ is given by (4-5). Note that we do not assume $\mathscr{L}$ to be natural here.

We now refine the notion of ordered sequences of Zimmermann subspaces as defined in [1], Sect. 5.

Definition 4.1. Let

$$
u^{(1)}, \ldots, u^{(r)}, \quad v^{(1)}, \ldots, v^{(m-r)}
$$

be an arbitrary basis of $\mathscr{L}$ with respect to $k$. A sequence $H_{1}, \ldots, H_{t}$ of subspaces in $\mathscr{H}^{\mathrm{UV}}$ is called ordered in $u$ with respect to the basis (4-10) if

1. $H_{1}, \ldots, H_{t}$ is ordered with respect to the basis (4-10), and

2. The parameters of every $H_{i}$ are contained in $\left(u^{(1)}, \ldots, u^{(r)}\right)$.

This notion will be very useful below when we define an "admissible" numerator $Z(\lambda, k, q)$. To this end, we first introduce sets $\mathscr{U}_{1}$ and $\mathscr{U}_{2}$ defined as follows.

1. $\mathscr{U}_{1}$ is the set of all pairs $(H, \mathscr{S})$ such that

a. $H \in \mathscr{H}^{\mathrm{uv}}, \mathscr{S} \subseteq \mathscr{S}_{0}$.

b. The complementary parameters of $H$ contain a basis of $\mathscr{S}$ with respect to $k$.

2. $\mathscr{U}_{2}$ is the set of pairs $(H, \mathscr{S})$ such that

a. $H \in \mathscr{H}^{\mathrm{IR}}, \mathscr{S} \subseteq \mathscr{S}_{0}$.

b. The parameters of $H$ are contained in a basis of $\mathscr{S}$ with respect to $k$.

A set of two maps

and

$$
\mathscr{U}_{1} \rightarrow \mathbf{Z}, \quad(H, \mathscr{S}) \rightarrow \delta(H, \mathscr{S})
$$

$$
\mathscr{U}_{2} \rightarrow \mathbf{Z}, \quad(H, \mathscr{S}) \rightarrow \rho(H, \mathscr{S})
$$

is called a degree set. In connection with the following definition it generalizes the notion of a UV-set as defined in [1].

We want to state the cutoff dependence of the integral (4-6). We assume that the numerator $Z(\lambda, k, q)$ is admissible with respect to a given degree set:

Definition 4.2. Suppose $\delta(H, \mathscr{S}), \rho(H, \mathscr{S})$ is a degree set. A nominator function $Z(\lambda, k, q)$ is then called admissible with respect to the degree set, if for every $\mathscr{S} \subseteq \mathscr{S}_{0}$ there is an $i \in I$, so that for every basis (4-10) of $\mathscr{L}$ with respect to $k$, where $u^{(1)}, \ldots, u^{(r)}$ is a basis of $\mathscr{S}$ with respect to $k$, the following conditions hold.

1. $M_{i j}(k, q) \equiv M_{i j}(u, q)$ for every $j \in J_{i}$, i.e. the polynomials $M_{i j}$ depend only on the basis of $\mathscr{S}$ and on the external momenta $q^{3}$.

2. For every sequence $H_{1}, \ldots, H_{t}$ of subspaces of $\mathscr{H}^{\mathrm{IR}}$ which is ordered in $u$ with respect to the basis (4-10), there exists $j \in J_{i}$, so that ${ }^{4}$

${ }^{3}$ If this holds for one basis of $\mathscr{S}$, it holds for any other basis of $\mathscr{S}$ also

${ }^{4}$ For the definition of $\operatorname{degr}_{u}$ see Appendix A 


$$
\underline{\operatorname{degr}}_{x_{g} \mid w_{g}} M_{i j} \geqq \rho\left(H_{g}, \mathscr{S}\right) \text { for all } g=1, \ldots, t .
$$

Here $\left(x_{g}\right)$ denotes the parameters of $H_{g}$ and $\left(x_{g}, w_{g}\right)=(u, v)$.

3. For every sequence $K_{1}, \ldots, K_{s}$ of subspaces of $\mathscr{H}^{\mathrm{UV}}$ which is ordered in $v$ with respect to the basis (4-10), there is a $l \in K_{i}$, so that

$$
\overline{\operatorname{degr}}_{y_{g} \mid z_{g}} C_{i l}-p_{i l} \leqq \delta\left(H_{g}, \mathscr{S}\right) \text { for all } g=1, \ldots, s,
$$

where $\left(y_{g}\right)$ denotes the parameters of $H_{g}$ and $\left(y_{g}, z_{g}\right)=(u, v)$.

The notion of an admissible numerator with respect to a given degree set generalizes the idea of an ultraviolet set of [1]. It enables us to control the ultraviolet as well as the infrared behavior of the integral (4-6).

We now define IR- and UV-divergence degrees for integrals of the form (4-6) with a numerator $Z(\lambda, k, q)$ which is admissible with respect to a given degree set (4-14). For $H \in \mathscr{H}^{\mathrm{UV}}$, parametrized by $(v)=\left(v_{1}, \ldots, v_{d}\right)$, a UV-divergence degree is defined by

$$
\begin{gathered}
\omega(H) \equiv \overline{\operatorname{degr}}_{H} \overline{\mathscr{I}}_{\lambda}=4 d+\delta(H)-\overline{\operatorname{degr}}_{v} E(k, q, \mu), \\
\delta(H)=\max _{\mathscr{S}} \delta(H, \mathscr{S}) .
\end{gathered}
$$

The maximum is over all $\mathscr{S}$ with $(H, \mathscr{S}) \in \mathscr{U}_{1}$. Furthermore, for a basis

$$
u_{1}=l_{i_{1}}, \ldots, u_{r}=l_{i_{r}}, \quad w_{1}=l_{j_{1}}, \ldots, w_{m-r}=l_{J_{m-r}},
$$

we define for every $H \in \mathscr{H}^{\mathrm{IR}}$, parametrized by $(u)=\left(u_{1}, \ldots, u_{r}\right)$, an IR-divergence degree by

$$
\begin{gathered}
r(H) \equiv \underline{\operatorname{degr}}_{H} \overline{\mathscr{I}}_{\lambda}=4 r+\rho(H)-\underline{\operatorname{degr}}_{u \mid w} E(k(u, w, q), q, \mu), \\
\rho(H)=\min _{\mathscr{S}} \rho(H, \mathscr{S}) .
\end{gathered}
$$

The minimum is over all $\mathscr{S}$ with $(H, \mathscr{S}) \in \mathscr{U}_{2}$.

The following theorem states the cutoff dependence of integrals (4-6) if a degree set is given with respect to which the numerator $Z(\lambda, k, q)$ is admissible.

Theorem 2. Auxiliary Theorem. Suppose the nominator function $Z(\lambda, k, q)$ of (4-6) is admissible with respect to a given degree set. Denote the corresponding divergence degrees by $\omega(H), H \in \mathscr{H}^{\mathrm{UV}}$, and by $r(H), H \in \mathscr{H}^{\mathrm{IR}}$. Suppose that for every $H \in \mathscr{H}^{\mathrm{IR}}$

$$
r(H)>0 .
$$

Then the integral $\overline{\mathscr{I}}_{\lambda}(q, \mu)$ exists for every finite $\hat{\lambda}$. Furthermore, there exist constants $K(\mu, q)>0$ and $c(\mu, q)>0$, so that for $\lambda>K(\mu, q)$,

$$
\overline{\mathscr{I}}_{\lambda}(q, \mu) \leqq c(\mu, q)
$$

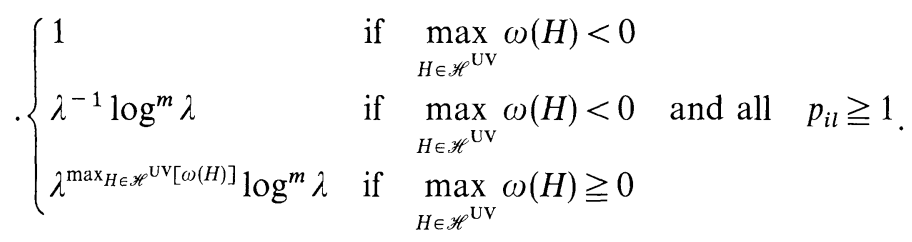


The proof of the auxiliary theorem is postponed to Sects. 7 and 8 .

We now state a corollary to the auxiliary theorem which will also be needed later on. Let

$$
\overline{\mathscr{J}}_{\lambda}(q, \mu)=\int^{\lambda, \mathscr{L}} d^{4} k_{1} \cdots d^{4} k_{m} \frac{|P(k, q)|}{E(k, q, \mu)},
$$

where $P(k, q)$ is a polynomial and $E(k, q, \mu)$ is given by (4-7). For such an integral divergence degrees are defined as follows. Let $\mathscr{H}^{\mathrm{UV}}$ and $\mathscr{H}^{\mathrm{IR}}$ as above. For $H \in \mathscr{H}^{\mathrm{UV}}$, parametrized by $(v)=\left(v_{1}, \ldots, v_{d}\right)$ and with complementary parameters $(z)=\left(z_{1}, \ldots, z_{m-d}\right)$, so that $k=k(v, z, q)$, we define a UV-divergence degree by

$$
\omega(H) \equiv \overline{\operatorname{degr}}_{H} \bar{J}_{\lambda}=4 d+\overline{\operatorname{degr}}_{v \mid z} P(k, q)-\overline{\operatorname{degr}}_{v \mid z} E(k, q, \mu) .
$$

An IR-divergence degree for $H \in \mathscr{H}^{\mathrm{IR}}$, parametrized by $(u)=\left(u_{1}, \ldots, u_{r}\right)$ and with complementary parameters $(w)=\left(w_{1}, \ldots, w_{m-r}\right)$, so that $k=k(u, w, q)$, is defined by

$$
r(H) \equiv \underline{\operatorname{degr}}_{H} \overline{\mathscr{J}}_{\lambda}=4 r+\underline{\operatorname{degr}}_{u \mid w} P(k, q)-\underline{\operatorname{degr}}_{u \mid w} E(k, q, \mu) .
$$

Corollary. Let

$$
\overline{\mathscr{J}}_{\lambda}(q, \mu)=\int^{\lambda, \mathscr{L}} d^{4} k_{1} \cdots d^{4} k_{m} \frac{|P(k, q)|}{E(k, q ; \mu)} .
$$

Suppose that for every $H \in \mathscr{H}^{\mathrm{IR}}$

$$
r(H)>0 .
$$

Then $\overline{\mathscr{J}}_{\lambda}(q, \mu)$ converges for every finite $\lambda$, and there exist constants $K(\mu, q)>0$ and $c(\mu, q)>0$, so that for $\lambda>K(\mu, q)$

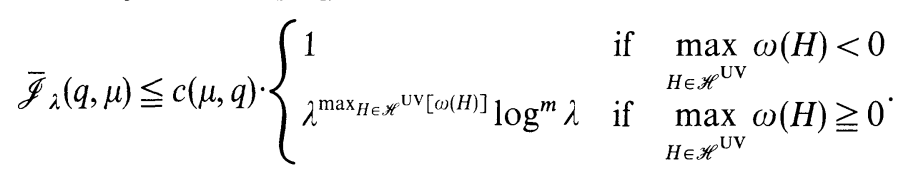

This is a direct consequence of the auxiliary theorem and is proved in Appendix B. Both the auxiliary theorem and its corollary will be used below to determine the cutoff dependence of the integrals into which the lattice Feynman integrals (3-12) are partitioned, as described at the end of Sect. 3.

\section{Bounds on the Numerator of a Lattice Feynman Integrand}

We now state an estimate for the numerator of a Feynman integrand (3-11) which allows an application of the auxiliary theorem of Sect. 4. To this end, let $\mathscr{L}$ again denote a natural set of line momenta and $\mathscr{M} \subseteq \mathscr{L}$.

Define a degree set $\mathscr{D}$ as the set of the following two maps.

1. For every $H \in \mathscr{H}^{\mathrm{UV}}$ and every $\mathscr{S} \subseteq \mathscr{M}$, set $\delta(H, \mathscr{S})=\overline{\operatorname{degr}}_{\hat{v}} V$, where $(v)$ is the parametrization of $H$.

2. For every $H \in \mathscr{H}^{\mathrm{IR}}$ and every $\mathscr{S} \subseteq \mathscr{M}$, set $\rho(H, \mathscr{S})=\operatorname{degr}_{\hat{u} \mid v} V$, where $(u)$ are the parameters and $(v)$ the complementary parameters of $\bar{H}$. 
Theorem 3. Let $V(k, q ; a) \in \mathscr{C}_{m_{0}}^{c}$ for some $m_{0} \in \mathbf{Z}$ and $(k a, q a)$ be bounded. Then $V$ can be estimated by

$$
|V(k, q ; a)-P(k, q)| \leqq a^{p} \sum_{b \in B} Z_{b}(k, q),
$$

where $B$ is a finite set and $p \in \mathbf{N}$. For every $b \in B, a^{p} Z_{b}(k, q)$ is a nominator function which is admissible with respect to the degree set $\mathscr{D}$. Furthermore, $P(k, q)=\lim _{a \rightarrow 0} V(k, q ; a)$. For every $H \in \mathscr{H}^{\mathrm{IR}}$, the inequality

$$
\underline{\operatorname{degr}}_{u \mid v} P \geqq \underline{\operatorname{degr}}_{\hat{u} \mid v} V
$$

holds, and for every $H \in \mathscr{H}^{\mathrm{Uv}}$, we have

$$
\overline{\operatorname{degr}}_{u \mid v} P \leqq \overline{\operatorname{degr}}_{\hat{u}} V,
$$

where $(u)$ denotes the parameters of $H$ and $(v)$ the complementary parameters.

Every function $a^{p} Z_{b}$ is of the form (4-8) with $\lambda$ replaced by $a^{-1}$, where all powers of $a$ are equal to $p$. If $P(k, q) \neq 0, p$ can be chosen to be 1 . If $P(k, q) \equiv 0, p$ is the largest natural number such that $\lim V(k, q ; a) / a^{p} \neq \equiv$ exists. Note that all the $\delta$ and $\rho$ of the degree set $\mathscr{D}$ are independent of subsets $\mathscr{S} \subseteq \mathscr{M}$ (cp. (3-4) and (4.12)f). The $\delta$ are independent also of the external momenta $q$. However, the IR-degrees are not so. The theorem looks like Theorem 3 in [1], the only difference being that we are now able to control also the IR-behavior.

The proof of Theorem 3 is postponed to Appendix C. We now start to prove the power counting theorem using this estimation and the auxiliary theorem.

\section{Proof of the Power Counting Theorem}

At first, the integration domain of (3-11) will be partitioned as indicated at the end of Sect. 3,

$$
\hat{I}(q ; \mu, a)=\sum_{J \subseteq\{1, \ldots, n\}} \sum_{z} \hat{I}_{J_{z}}(q ; \mu, a),
$$

where for every $J$ the sum over $z$ is finite, and for every sector $J, z=\left(z_{i} \in \mathbf{Z}^{4} \mid i \in J\right)$,

$$
\begin{aligned}
\hat{I}_{J_{z}}(q ; \mu, a)= & \int_{-\pi / a}^{\pi / a} d^{4} k_{1} \cdots d^{4} k_{m} \frac{V(k, q ; \mu, a)}{\prod_{i=1}^{n}\left(\frac{\eta_{i}\left(l_{i} a\right)}{a^{2}}+\mu_{i}^{2}\right)} \\
& \cdot\left(\prod_{i \in J} \Theta\left(\frac{\pi}{a} \varepsilon-\left\|l_{i}-\frac{2 \pi}{a} z_{i}\right\|\right)\right) \prod_{i \notin J} \Theta_{\varepsilon}\left(l_{i}\right) .
\end{aligned}
$$

Here, $\Theta$ is the Heaviside step function, $\Theta(x)=1$ for $x \geqq 0$ and $\Theta(x)=0$ for $x<0$, and

$$
\Theta_{\varepsilon}(l)= \begin{cases}0 & \text { if }\left\|l-\frac{2 \pi}{a} z\right\|<\frac{\pi}{a} \varepsilon \text { for some } z \in \mathbf{Z}^{4} \\ 1 & \text { otherwise, }\end{cases}
$$

and $\varepsilon$ is a positive constant. If $\varepsilon>0$ is small enough, for every $J, z$ one can find a 
translation $k_{j} \rightarrow k_{j}+(2 \pi / a) \delta_{j}, j=1, \ldots, m$, so that

$$
l_{i} \rightarrow l_{i}+\frac{2 \pi}{a} z_{i} \text { for all } i \in J
$$

This is a direct consequence of the naturalness of line momenta ([1], Appendix D). Hence

$$
\hat{I}_{J_{z}}(q ; \mu, a)=\int_{\sigma_{J}} d^{4} k_{1} \cdots d^{4} k_{m} \frac{V(k, q ; a)}{\prod_{i=1}^{n}\left(\frac{\eta_{i}\left(l_{i} a\right)}{a^{2}}+\mu_{i}^{2}\right)}\left(\prod_{i \in J} \Theta\left(\frac{\pi}{a} \varepsilon-\left\|l_{i}\right\|\right)\right) \cdot \prod_{i \neq J} \Theta_{\varepsilon}\left(l_{i}\right),
$$

where

$$
\begin{aligned}
\sigma_{J} & =\left\{\left(k_{1}, \ldots, k_{m}\right) \in \mathbf{R}^{4 m} \mid-\frac{\pi}{a}-\frac{2 \pi}{a}\left(\delta_{j}\right)_{i} \leqq\left(k_{j}\right)_{i}\right. \\
& \left.\leqq \frac{\pi}{a}-\frac{2 \pi}{a}\left(\delta_{j}\right)_{i}, \quad j=1, \ldots, m ; i=1, \ldots, 4\right\} .
\end{aligned}
$$

Using Theorem 3, we write $V(k, q ; a)=P(k, q)+R(k, q ; a)$ and $\hat{I}_{J_{z}}=\hat{I}_{J_{z}}^{0}+\hat{I}_{J_{z}}^{R}$, where

$$
\hat{I}_{J_{z}}^{0}=\int_{\sigma_{J}} d^{4} k_{1} \cdots d^{4} k_{m} \frac{P(k, q)}{\prod_{i=1}^{n}\left(\frac{\eta_{i}\left(l_{i} a\right)}{a^{2}}+\mu_{i}^{2}\right)}\left(\prod_{i \in J} \Theta\left(\frac{\pi}{a} \varepsilon-\left\|l_{i}\right\|\right)\right) \cdot \prod_{i \notin J} \Theta_{\varepsilon}\left(l_{i}\right),
$$

and

$$
\hat{I}_{J_{z}}^{R}=\int_{\sigma_{J}} d^{4} k_{1} \cdots d^{4} k_{m} \frac{R(k, q ; a)}{\prod_{i=1}^{n}\left(\frac{\eta_{i}\left(l_{i} a\right)}{a^{2}}+\mu_{i}^{2}\right)}\left(\prod_{i \in J} \Theta\left(\frac{\pi}{a} \varepsilon-\left\|l_{i}\right\|\right)\right) \cdot \prod_{i \notin J} \Theta_{\varepsilon}\left(l_{i}\right) .
$$

$R(k, q ; a)$ admits an estimate of the form

$$
|R(k, q ; a)| \leqq a^{p} \sum_{b \in B} Z_{b}(k, q),
$$

where $p \in \mathbf{N}, B$ is a finite set, and for every $b \in B$ the function $a^{p} Z_{b}(k, q)$ is a nominator function which is admissible with respect to the degree set $\mathscr{D}$, defined at the beginning of Sect. 5 .

As an elementary property of the propagators, for small enough $\varepsilon$ there are constants $\alpha$ and $\gamma$, so that

$$
\frac{1}{\frac{\eta_{i}\left(l_{i} a\right)}{a^{2}}+\mu_{i}^{2}} \leqq \frac{\alpha}{l_{i}^{2}+\mu_{i}^{2}}
$$

for all $\left\|l_{i}\right\|<(\pi / a) \varepsilon$, and

$$
\frac{1}{\frac{\eta_{i}\left(l_{i} a\right)}{a^{2}}+\mu_{i}^{2}} \leqq \gamma a^{2}
$$


whenever $\left\|l_{i}-(2 \pi / a) z\right\| \geqq(\pi / a) \varepsilon$ for all $z \in \mathbf{Z}^{4}$. Let $h$ be the number of elements of $J$. Using the bounds (6-10), (6-11), we get the estimates

$$
\left|\hat{I}_{J_{z}}^{0}(q ; \mu, a)\right| \leqq \bar{I}_{J_{z}}^{0}(q, \mu, a)=\alpha^{h}\left(\gamma a^{2}\right)^{n-h} \int_{\kappa_{J}} d^{4} k_{1} \cdots d^{4} k_{m} \frac{|P(k, q)|}{\prod_{i \in J}\left(l_{i}^{2}+\mu_{i}^{2}\right)},
$$

and

where

$$
\left|\hat{I}_{J_{z}}^{R}(q ; \mu, a)\right| \leqq \sum_{b \in B} \bar{I}_{J_{z}}^{(b)}(q, \mu, a)
$$

$$
\begin{aligned}
\bar{I}_{J_{z}}^{(b)}(q, \mu, a) & =\alpha^{h}\left(\gamma a^{2}\right)^{n-h} \int_{\kappa_{J}} d^{4} k_{1} \cdots d^{4} k_{m} \frac{a^{p} Z_{b}(k, q)}{\prod_{i \in J}\left(l_{i}^{2}+\mu_{i}^{2}\right)}, \\
\kappa_{J} & =\left\{\left(k_{1}, \ldots, k_{m}\right) \in \mathbf{R}^{4 m} \mid\left\|l_{j}\right\| \leqq \frac{\delta}{a} \text { for all } l_{j} \in \mathscr{L}_{J}\right\}, \\
\mathscr{L}_{J} & =\left\{l_{j} \mid j \in J\right\} \cup\left\{k_{1}, \ldots, k_{m}\right\} \subseteq \mathscr{L}, \\
\delta & =\max _{i=1, \ldots, m}\left(\pi \varepsilon, 4 \pi\left(1+\frac{\left\|\delta_{i}\right\|}{2}\right)\right) .
\end{aligned}
$$

To every integral in (6-12) or (6-13) we now apply the auxiliary theorem or its corollary, respectively. All the integrals are of the form needed, $\lambda$ being replaced by $\delta / a$ and $\mathscr{L}$ by $\mathscr{L}_{J}$. The corresponding sets of subspaces $\mathscr{H}_{J}^{\mathrm{UV}}$ and $\mathscr{H}_{J}^{\mathrm{IR}}$ are defined by basis of $\mathscr{L}_{J}$ with respect to $k$. By (6-15), $\mathscr{H}_{J}^{\mathrm{UV}} \subseteq \mathscr{H}^{\mathrm{UV}}$ and $\mathscr{H}_{J}^{\mathrm{IR}} \subseteq \mathscr{H}^{\mathrm{IR}}$.

We first consider the integrals $\bar{I}_{J_{z}}^{(b)}$. Every integral in (6-13) satisfies the conditions to apply the auxiliary theorem with the degree set

$$
\rho(H, \mathscr{S})=\underline{\operatorname{degr}}_{\hat{u} \mid w} V, \quad \text { and } \quad \delta(H, \mathscr{S})=\overline{\operatorname{degr}}_{\hat{0}} V
$$

for $H \in \mathscr{H}_{J}^{\mathrm{IR}}$ with parametrization $(u)=\left(u^{(1)}, \ldots, u^{(r)}\right)$ with respect to a basis $(u, w)$ of $\mathscr{L}_{J}$ and for $H \in \mathscr{H}_{J}^{\mathrm{UV}}$ with parametrization $(v)$, respectively. Remember that the $\delta$ and $\rho$ are independent of the subsets $\mathscr{S} \subseteq \mathscr{M}$. In the notation (4-20), for every $H \in \mathscr{H}_{J}^{\mathrm{IR}}$, parametrized by $(u)=\left(u^{(1)}, \ldots, u^{(r)}\right)$ and with complementary parameters $(w)$,

$$
\begin{aligned}
\underline{\operatorname{degr}}_{H} \bar{I}_{J_{z}}^{(b)}(q, \mu, a) & =4 r+\rho(H)-\underline{\operatorname{degr}}_{u \mid w} \prod_{i \in J}\left(l_{i}^{2}+\mu_{i}^{2}\right) \\
& =\left[4 r+\underline{\operatorname{degr}}_{\hat{u} \mid w} V-\underline{\operatorname{degr}}_{\hat{u} \mid w} C\right]+\underline{\operatorname{degr}}_{u \mid w} \prod_{i \neq J}\left(l_{i}^{2}+\mu_{i}^{2}\right)>0,
\end{aligned}
$$

where we have used (3-6). Hence, using the auxiliary theorem, all integrals in (6-13) are convergent, for every finite lattice spacing $a$. Furthermore, for every $H \in \mathscr{H}_{J}^{\mathrm{UV}}$, with parametrization $(v)=\left(v_{1}, \ldots, v_{d}\right)$

$$
\begin{aligned}
\overline{\operatorname{degr}}_{H} \bar{I}_{J_{z}}^{(b)}(q, \mu, a) & =4 d+\delta(H)-\overline{\operatorname{degr}}_{v} \prod_{i \in J}\left(l_{i}^{2}+\mu_{i}^{2}\right) \\
& =\left[4 d+\overline{\operatorname{degr}}_{\hat{v}} V-\overline{\operatorname{degr}}_{\hat{0}} C\right]+\overline{\operatorname{degr}}_{v} \prod_{i \neq J}\left(l_{i}^{2}+\mu_{i}^{2}\right) \\
& <2(n-h),
\end{aligned}
$$


where we have used (3-7), i.e.

$$
\overline{\operatorname{degr}}_{H} \bar{I}_{J_{z}}^{(b)}(q, \mu, a) \leqq 2(n-h)-1 \text { for all } H \in \mathscr{H}_{J}^{\mathrm{UV}} .
$$

Using the auxiliary theorem, there exist positive $K$ and $c$, so that for all $a<K^{-1}$,

a. if $n-h>0, \quad \bar{I}_{J_{z}}^{(b)}(q, \mu, a) \leqq c\left(a^{2}\right)^{n-h} a^{-[2(n-h)-1]} \log ^{m} a=c a \log ^{m} a$.

b. if $n-h=0, \quad \bar{I}_{J_{z}}^{(b)}(q, \mu, a) \leqq c a \log ^{m} a \quad$ (because of $p \geqq 1$ ).

This means that the remainder $\hat{I}_{J_{z}}^{R}$ does not contribute in the continuum limit. If $P(k, q) \equiv 0$, all $\hat{I}_{J_{z}}^{0}$ vanish, and the proof of the power counting theorem is complete.

Thus, let us assume that $P(k, q) \neq 0$. For every $H \in \mathscr{H}_{J}^{\mathrm{IR}}$ with parametrization $(u)=\left(u_{1}, \ldots, u_{r}\right)$ (and complementary variables $(w)=\left(w_{1}, \ldots, w_{m-r}\right)$ with respect to a basis $(u, w)$ of $\left.\mathscr{L}_{J}\right)$,

$$
\underline{\operatorname{degr}}_{u \mid w} P \geqq \underline{\operatorname{degr}}_{\hat{u} \mid w} V .
$$

Hence, in the notation of (4-26), using (3-6),

$$
\begin{aligned}
& \underline{\operatorname{degr}}_{H} \bar{I}_{J_{z}}^{0}(q, \mu, a)=4 r+\underline{\operatorname{degr}}_{u \mid w} P(k, q)-\underline{\operatorname{degr}}_{u \mid w} \prod_{i \in J}\left(l_{i}^{2}+\mu_{i}^{2}\right) \\
& \geqq\left[4 r+\left.\underline{\operatorname{degr}_{\hat{u} \mid w} V-\underline{\operatorname{degr}}} \underline{u}\right|_{\mid w} C\right]+\underline{\operatorname{degr}_{u \mid w}} \prod_{i \notin J}\left(l_{i}^{2}+\mu_{i}^{2}\right)>0 .
\end{aligned}
$$

Hence, by the corollary to the auxiliary theorem, $\bar{I}_{J_{z}}^{0}(q, \mu, a)$ is absolutely convergent for evey finite $a>0$. If in addition (3-7) holds, then for every $H \in \mathscr{H}_{J}^{\mathrm{UV}}$ with parametrization $(v)=\left(v_{1}, \ldots, v_{d}\right),(z)$ being the complementary parameters of $H$,

$$
\begin{aligned}
\overline{\operatorname{degr}}_{H} \bar{I}_{J_{z}}^{0}(q, \mu, a) & =4 d+\overline{\operatorname{degr}}_{v \mid z} P(k, q)-\overline{\operatorname{degr}}_{v \mid z} \prod_{i \in J}\left(l_{i}^{2}+\mu_{i}^{2}\right) \\
& \leqq\left[4 d+\overline{\operatorname{degr}}_{\hat{v}} V-\overline{\operatorname{degr}}_{\hat{0}} C\right]+\overline{\operatorname{degr}}_{v} \prod_{l \notin J}\left(l_{i}^{2}+\mu_{i}^{2}\right)<2(n-h),
\end{aligned}
$$

hence

$$
\overline{\operatorname{degr}}_{H} \bar{I}_{J_{z}}^{0}(q, \mu, a) \leqq 2(n-h)-1
$$

for every $H \in \mathscr{H}_{J}^{\mathrm{UV}}$. Again applying the corollary to the auxiliary theorem, there are constants $K$ and $c$, so that for all $a<1 / K$,

$$
\begin{aligned}
& \text { a. if } n-h>0, \quad \bar{I}_{J_{z}}^{0}(q, \mu, a) \leqq c\left(a^{2}\right)^{n-h} \cdot a^{-[2(n-h)-1]} \log ^{m} a=c a \log ^{m} a, \\
& \text { b. if } n-h=0, \quad \bar{I}_{J_{z}}^{0}(q, \mu, a) \leqq c .
\end{aligned}
$$

We thus see that the continuum limit of $\hat{I}(q ; \mu, a)$ exists. As in the massive case, by the naturalness of line momenta, there is only one sector which contributes in this limit, given by $J=\{1, \ldots, n\}$ and $z=0$. Using the dominated convergence theorem of Lebesgue, we get

$$
\lim _{a \rightarrow 0} \hat{I}(q ; \mu, a)=\int_{-\infty}^{\infty} d^{4} k_{1} \cdots d^{4} k_{m} \frac{P(k, q)}{\prod_{i=1}^{n}\left(l_{i}^{2}+\mu_{i}^{2}\right)} .
$$

This completely proves the power counting theorem for Feynman integrals with massless propagators. 


\section{A Lemma about IR-Behavior}

To prove the auxiliary theorem we need a statement about the IR-behavior of generalized continuum Feynman integrals. The integrals considered in this section are of the general form

$$
\bar{J}=\int_{l_{i}^{2} \leqq 1 \text { in } \mathscr{V}} d^{4} u_{1} \cdots d^{4} u_{r} \frac{\min _{i \in I}\left|M_{i}(u)\right|}{\prod_{\mathscr{V}}\left(l_{j}(u)^{2}\right)^{n_{J}}},
$$

where $u_{1}, \ldots, u_{r} \in \mathbf{R}^{4}, \mathscr{W}=\left\{l_{j}(u) \mid j=1, \ldots, w\right\}$, and the $l_{j}$ 's are linear combinations of $u_{1}, \ldots, u_{r}$ in such a way that $\operatorname{rank}_{u} \mathscr{W}=r$. Without loss of generality we assume the $l_{j}^{2}$ to be mutually different. $\mathscr{V}$ is a subset of $\mathscr{W}$, and for $l_{j} \in \mathscr{V}$ we assume $n_{j} \in \mathbf{N}$. $I$ is a finite set, and all $M_{i}$ are polynomials in the components of $u_{1}, \ldots, u_{r}$. The integration domain consists of those $u$ satisfying $l_{i}^{2}(u) \leqq 1$ for all $l_{i} \in \mathscr{W}$.

A set $\mathscr{H}^{\mathrm{IR}}$ of equivalence classes of affine subspaces of $\left(u_{1}, \ldots, u_{r}\right)$ is defined as in Sect. 3 (with $\mathscr{W}$ for $\mathscr{L}, \mathscr{V}$ for $\mathscr{M}$ and $u$ instead of $k$ ). To make a statement about the convergence of (7-1), we introduce the notion of an IR-set for the family of polynomials $M_{i}{ }^{5}$.

Definition 7.1. The set $\left\{\rho(H) \mid H \in \mathscr{H}^{\mathrm{IR}}\right\}$ is called an infrared-set (IR-set), if

1. $\rho(H) \in \mathbf{Z}$ for all $H \in \mathscr{H}^{\mathrm{IR}}$.

2. For any basis $\left(x_{1}, \ldots, x_{r}\right)$ of $\mathscr{W}$ with respect to $u$ and any sequence $H_{1}, \ldots, H_{t}$ of subspaces in $\mathscr{H}^{\mathrm{IR}}$ which is ordered with respect to this basis, there is an $i \in I$ so that

$$
\underline{\operatorname{degr}}_{z_{j} \mid w_{j}} M_{i} \geqq \rho\left(H_{j}\right) \text { for all } j=1, \ldots, t,
$$

where $\left(z_{j}\right)$ are the parameters of $H_{j}$ and $\left(z_{j}, w_{j}\right)=\left(x_{1}, \ldots, x_{r}\right), j=1, \ldots, t$.

The integral (7-1) does not depend on external momenta. In (7-2), no momenta are fixed (in the sense of Appendix A). For this reason we will omit the complementary variables throughout this section (and only here) once a basis is given, i.e. we write

$$
\underline{\operatorname{degr}}_{z_{j}} M_{i} \equiv \underline{\operatorname{degr}}_{z_{J} \mid w_{J}} M_{i}
$$

Depending on an IR-set, we define divergence degrees of (7-1) for arbitrary $H \in \mathscr{H}^{\mathrm{IR}}$ as follows. Let

$$
z_{1}=l_{i_{1}}, \ldots, z_{s}=l_{i_{s}}, \quad w_{1}=l_{j_{1}}, \ldots, w_{r-s}=l_{j_{r-s}}
$$

be a basis of $\mathscr{W}$ with respect to $u$, where $l_{i_{1}}, \ldots, l_{i_{s}} \in \mathscr{V}$. Then for $H \in \mathscr{H}^{\mathrm{IR}}$, parametrized by $(z)=\left(z_{1}, \ldots, z_{s}\right)$, we define an IR-degree of (7-1) by

$$
r(H)=4 s+\rho(H)-\underline{\operatorname{degr}}_{z} \prod_{\mathscr{V}}\left(l_{j}^{2}(u(z, w))\right)^{n_{j}} .
$$

Lemma 7.1. IR-Lemma. Assume that an IR-set is given. Let $\left\{r(H) \mid H \in \mathscr{H}^{\mathrm{IR}}\right\}$ be the corresponding set of IR-divergence degrees. Suppose that for every $H \in \mathscr{H}^{\mathrm{IR}}$,

$$
r(H)>0 .
$$

${ }^{5} \mathrm{cp}$. the notion of a UV-set in [1] 
Then the integral

$$
\overline{\mathscr{J}}=\int_{l_{i}^{2} \leqq 1 \text { in } \mathscr{W}} d^{4} u_{1} \cdots d^{4} u_{r} \frac{\min _{i \in I}\left|M_{i}(u)\right|}{\prod_{\mathscr{V}}\left(l_{j}(u)^{2}\right)^{n_{j}}}
$$

is convergent.

We now prove the lemma by induction on the number $r$ of four-dimensional integrations. The idea of proof is similar to that of the auxiliary power counting theorem of [1]. The integration domain is decomposed into various parts and the resulting section integrals are split appropriately with the aim to do one integration in an elementary way and to apply the induction hypothesis to the other integrations.

The case $r=0$ is trivial. Hence let $r \geqq 1$. For every $\xi=1, \ldots, w$ we define a sector $X_{\xi} \subset \mathbf{R}^{4 r}$ as the set of those $u$ satisfying

$$
l_{i}^{2}(u) \leqq l_{\xi}^{2}(u) \leqq 1 \text { for all } i=1, \ldots, w .
$$

Next, we make a linear non-singular transformation

$$
t_{i}=\sum_{j=1}^{r}\left(A_{\xi}\right)_{i j} u_{j} ; \quad\left(A_{\xi}\right)_{i j} \in \mathbf{R} ; \quad i, j=1, \ldots, r
$$

so that

$$
t_{1}=l_{\xi}(u) \text { and } \operatorname{det}\left(A_{\xi}\right)=1 .
$$

Define $\mathscr{W}_{\xi}=\mathscr{W} \backslash\left\{l_{\xi}\right\}$ and $\mathscr{V}_{\xi}=\mathscr{V} \backslash\left\{l_{\xi}\right\}$. For every $\xi$ and $\mathscr{S} \subseteq \mathscr{V}_{\xi}$ we choose a basis

$$
z_{1}, \ldots, z_{s}, \quad v_{1}, \ldots, v_{r-s-1}
$$

of $\mathscr{W}_{\xi}$ with respect to $\left(t_{2}, \ldots, t_{r}\right)$ such that $z_{1}, \ldots, z_{s}$ is a basis of $\mathscr{S}$ with respect to $\left(t_{2}, \ldots, t_{r}\right)$. Then $v_{1}, \ldots, v_{r-s-1} \in \mathscr{W}_{\xi} \backslash \mathscr{S}$ and

$$
u=u\left(z, v, t_{1}\right) \equiv f_{\xi \mathscr{S}}\left(z, v, t_{1}\right)
$$

is a linear function. Every $l_{i} \in \mathscr{S}$ has a $(\xi, \mathscr{S}$-dependent) representation

$$
l_{i}\left(z, t_{1}\right)=\sum_{j=1}^{s} c_{i j} z_{j}+d_{i} t_{1} .
$$

Let $\mathscr{H}_{\mathscr{V}}$ be the set of all $H \in \mathscr{H}^{\mathrm{IR}}$ which are parametrized by a basis of $\mathscr{V}$ with respect to $\left(u_{1}, \ldots, u_{r}\right)$. Set

$$
\Delta=\min _{H \in \mathscr{H}_{\mathscr{r}}} \rho(H) .
$$

For every $\xi, \mathscr{S} \subseteq \mathscr{V}_{\xi}$, let $H_{1}, \ldots, H_{t}$ be an arbitrary, ordered sequence of spaces of $\mathscr{H}^{\text {IR }}$, so that

a. for $j=1, \ldots, t-1, H_{j}$ is parametrized by $\left(k_{j}\right) \subseteq \mathscr{S}$.

b. $H_{t}$ is parametrized by a basis $\left(k_{t}\right)$ of $\mathscr{V}(\supseteq \mathscr{S})$ with respect to $\left(u_{1}, \ldots, u_{r}\right)$.

By assumption, for every such sequence there is an $i \in I$, so that

$$
\underline{\operatorname{degr}}_{k_{j}} M_{i} \geqq \rho\left(H_{j}\right) \text { for all } j=1, \ldots, t .
$$


The set of all these $i \in I$ is denoted by $I(\xi, \mathscr{S})$.

We now give an appropriate estimation for the integral $\bar{J}$ (Lemma 7.2) which allows us to apply the hypothesis of induction (Lemma 7.3).

Lemma 7.2. For any $0<\varepsilon<1$, the integral (7-1) admits an estimate

$$
\overline{\mathscr{J}} \leqq \sum_{\xi=1}^{w} \sum_{\mathscr{S} \subseteq \mathscr{V}_{\xi}} \sum_{y \in Y(\xi, \mathscr{S})} \int_{t_{1}^{2} \leqq 1} d^{4} t_{1}\left\|t_{1}\right\|^{\eta \cdot} \cdot \overline{\mathscr{J}}_{\xi, \mathscr{S}, y}^{\mathrm{int}}\left(t_{1}\right)
$$

where $Y(\xi, \mathscr{S})$ are finite sets, $\eta>-4$ and

$$
\begin{aligned}
\overline{\mathscr{J}}_{\xi, \mathscr{S}, y}^{\mathrm{int}}\left(t_{1}\right)= & \int_{l_{1}^{2}\left(z^{\prime}, t_{1}^{\prime}\right) \leq \delta^{2} \text { in } \mathscr{S}} d^{4} z_{1}^{\prime} \cdots d^{4} z_{s}^{\prime} \frac{1}{\prod_{\mathscr{S}}\left(l_{j}^{2}\left(z^{\prime}, t_{1}^{\prime}\right)\right)^{n_{j}}} . \\
& \cdot \int_{\Omega^{\prime}(\xi, \mathscr{S})} d^{4} v_{1}^{\prime} \cdots d^{4} v_{r-s-1}^{\prime} \frac{\min _{i \in[\xi, \mathscr{S})}\left|P_{i y}\left(v^{\prime}, t_{1}^{\prime}\right) \cdot T_{i y}\left(z^{\prime}\right)\right|}{\prod_{\mathscr{V} \backslash \mathscr{S}}\left(l_{j}^{2}\left(u\left(z^{\prime}, v^{\prime}, t_{1}^{\prime}\right)\right)\right)^{n_{j}}},
\end{aligned}
$$

where $t_{1}^{\prime}=t_{1} /\left\|t_{1}\right\|$,

$$
\Omega^{\prime}(\xi, \mathscr{S})=\left\{\left(v_{1}^{\prime}, \ldots, v_{r-s-1}^{\prime}\right) \mid \begin{array}{l}
1 \geqq l_{i}^{2}\left(u\left(z^{\prime}, v^{\prime}, t_{1}^{\prime}\right)\right) \geqq \varepsilon^{2} \text { if } l_{i} \in \mathscr{V}_{\xi} \backslash \mathscr{S} \\
1 \geqq l_{i}^{2}\left(u\left(z^{\prime}, v^{\prime}, t_{1}^{\prime}\right)\right) \text { if } l_{i} \in \mathscr{W}_{\xi} \backslash \mathscr{V}_{\xi}
\end{array}\right\},
$$

and for every $\xi, \mathscr{S}, u\left(z^{\prime}, v^{\prime}, t_{1}^{\prime}\right) \equiv f_{\xi \mathscr{Y}}\left(z^{\prime}, v^{\prime}, t_{1}^{\prime}\right)$, where $f_{\xi \mathscr{S}}$ is defined in $(7-8 b)$. For every $i \in I(\xi, \mathscr{S})$ and every $y \in Y(\xi, \mathscr{S}), P_{i y}$ and $T_{i y}$ are polynomials, and for any affine subspace of the $\left(z^{\prime}\right)$-variables, parametrized by $(\tilde{z})$ say, we have

$$
\operatorname{degr}_{\tilde{z}} T_{i y}\left(z^{\prime}\right) \geqq \underline{\operatorname{degr}_{\tilde{z}}} M_{i}\left(u\left(z^{\prime}, v^{\prime}, t_{1}^{\prime}\right)\right) .
$$

Proof. Applying the transformations (7-7) and (7-8) to $\overline{\mathscr{J}}$ for every $\xi$ and $\mathscr{S}$ and noticing that

$$
\bigcup_{\xi=1}^{w} X_{\xi}=\left\{\left(u_{1}, \ldots, u_{r}\right) \in \mathbf{R}^{4 r} \mid l_{j}^{2}(u) \leqq 1 \text { for all } l_{j} \in \mathscr{W}\right\},
$$

we get

where

$$
\overline{\mathscr{J}} \leqq \sum_{\xi=1}^{w} \sum_{\mathscr{S} \subseteq \mathscr{V}_{\xi}} \overline{\mathscr{J}}_{\xi \mathscr{P}}
$$

and

$$
\begin{aligned}
\overline{\mathscr{J}}_{\xi, \mathscr{S}}= & \int_{t_{1}^{2} \leqq 1} d^{4} t_{1} \int_{l_{i}^{2} \leqq \varepsilon^{2} t_{1}^{2} \text { in } \mathscr{S}} d^{4} z_{1} \cdots d^{4} z_{s} \frac{1}{\prod_{\mathscr{S}}\left(l_{j}^{2}\right)^{n_{j}}} . \\
& \cdot \int_{\Omega\left(\xi_{,}, \mathscr{G}\right)} d^{4} v_{1} \cdots d^{4} v_{r-s-1} \frac{\min _{i \in I}\left|M_{i}\left(u\left(z, v, t_{1}\right)\right)\right|}{\prod_{\mathscr{W} \backslash \mathscr{\mathscr { S }}}\left(l_{j}^{2}\right)^{n_{J}}},
\end{aligned}
$$

$$
\Omega(\xi, \mathscr{S})=\left\{\left(v_{1}, \ldots, v_{r-s-1}\right) \mid \begin{array}{l}
t_{1}^{2} \geqq l_{i}^{2}\left(u\left(z, v, t_{1}\right)\right) \geqq \varepsilon^{2} t_{1}^{2} \text { if } l_{i} \in \mathscr{V}_{\xi} \backslash \mathscr{S} \\
t_{1}^{2} \geqq l_{i}^{2}\left(u\left(z, v, t_{1}\right)\right) \text { if } l_{i} \in \mathscr{W}_{\xi} \backslash \mathscr{V}_{\xi}
\end{array}\right\} .
$$

Here we have used (7-9) for every $l_{i} \in \mathscr{S}$. We now decompose the polynomials in the numerator into linearly independent homogeneous polynomials $M_{i \alpha}$ of the 
order $\alpha$ in $u$

$$
M_{i}(u)=\sum_{\alpha \geqq 0} M_{i \alpha}(u)
$$

and furthermore, every $M_{i \alpha}$ is decomposed into linearly independent homogeneous polynomials $T_{\beta}(z)$ in $z$,

$$
M_{i \alpha}(u)=\sum_{\beta} P_{i \alpha \beta}\left(v, t_{1}\right) T_{\beta}(z), \quad P_{i \alpha \beta}\left(v, t_{1}\right) \neq \equiv
$$

in such a way that the polynomials $P_{i \alpha \beta}$ for fixed $i, \alpha$ are linearly independent. Lemma A.1 in the Appendix states that this is always possible. Hence

$$
M_{i}(u)=\sum_{\alpha \beta} P_{i \alpha \beta}\left(v, t_{1}\right) T_{\beta}(z)
$$

Using the linear independence of the $M_{i \alpha}$ for fixed $i$ and Lemma A.1 again, for any affine subspace of the $(z)$-variables, parametrized by $(\tilde{z})$ say, we get

$$
\underline{\operatorname{degr}_{\tilde{z}}} T_{\beta}(z) \geqq \underline{\operatorname{degr}}{ }_{\tilde{z}} M_{i}\left(u\left(z, v, t_{1}\right)\right),
$$

for every $T_{\beta}$ in (7-19c). Hence, for every $\xi, \mathscr{S}$,

$$
\begin{aligned}
\overline{\mathscr{J}}_{\xi, \mathscr{S}} \leqq & \sum_{\left(\alpha_{\imath}, \beta_{\imath}\right) \text { for all }} \int_{i \in I} d_{t_{1}^{2} \leqq 1} d_{1} \int_{l_{i}^{2} \leqq \varepsilon^{2} t_{1}^{2} \text { in } \mathscr{S}} d^{4} z_{1} \cdots d^{4} z_{s} \frac{1}{\prod_{\mathscr{S}}\left(l_{j}^{2}\left(z, t_{1}\right)\right)^{n_{j}}} . \\
& \cdot \int_{\Omega(\xi, \mathscr{S})} d^{4} v_{1} \cdots d^{4} v_{r-s-1} \frac{\min _{i \in I(\xi, \mathscr{S})}\left|P_{i \alpha_{\imath} \beta_{\imath}}\left(v, t_{1}\right) T_{\beta_{i}}(z)\right|}{\prod_{\mathscr{V} \backslash \mathscr{S}}\left(l_{j}^{2}\left(u\left(z, v, t_{1}\right)\right)^{n_{J}}\right.},
\end{aligned}
$$

where the minimum has been restricted to $I(\xi, \mathscr{S})$. Let $r_{i}=\operatorname{degr}_{u} M_{i}(u)$ for every $i \in I(\xi, \mathscr{S})$. By definition $(7-10)$ of $\Delta$,

Substituting

$$
r_{i}-\Delta \geqq 0
$$

$$
\left(z_{1}, \ldots, z_{s}\right)=\left(z_{1}^{\prime}, \ldots, z_{s}^{\prime}\right) \cdot\left\|t_{1}\right\|, \quad\left(v_{1}, \ldots, v_{r-s-1}\right)=\left(v_{1}^{\prime}, \ldots, v_{r-s-1}^{\prime}\right) \cdot\left\|t_{1}\right\|,
$$

and writing $t_{1}^{\prime}=t_{1} /\left\|t_{1}\right\|$, we get

$$
\begin{aligned}
\overline{\mathscr{J}}_{\xi, \mathscr{S}} \leqq & \sum_{y \in Y(\xi, \mathscr{S})} \int_{t_{1}^{2} \leqq 1} d^{4} t_{1}\left\|t_{1}\right\|^{\eta} \int_{l_{i}^{2} \leqq \varepsilon^{2} \text { in } \mathscr{S}} d^{4} z_{1}^{\prime} \cdots d^{4} z_{s}^{\prime} \frac{1}{\prod_{\mathscr{S}}\left(l_{j}^{2}\left(z^{\prime}, t_{1}^{\prime}\right)\right)^{n_{j}}} \\
& \cdot \int_{\Omega^{\prime}(\xi, \mathscr{S})} d^{4} v_{1}^{\prime} \cdots d^{4} v_{r-s-1}^{\prime} \frac{\min _{t \in I(\xi, \mathscr{Y})}\left\|t_{1}\right\|^{r_{i}-\Delta \cdot}\left|P_{i y}\left(v^{\prime}, t_{1}^{\prime}\right) T_{i y}\left(z^{\prime}\right)\right|}{\prod_{\mathscr{L} \backslash \mathscr{S}}\left(l_{j}^{2}\left(u\left(z^{\prime}, v^{\prime}, t_{1}^{\prime}\right)\right)\right)^{n_{j}}},
\end{aligned}
$$

where we have collected indices, and $\eta=4(r-1)-\underline{\operatorname{degr}}_{u} \prod\left(l_{j}^{2}(u)\right)^{n_{J}}+\Delta$. Choose any $H \in \mathscr{H}_{\mathscr{V}}$, parametrized by $(w)=\left(w_{1}, \ldots, w_{b}\right)$ say, such that $\rho(H)=\Delta$. Then

$$
\eta=4(r-1)-\underline{\operatorname{degr}} u \prod_{\mathscr{V}}\left(l_{j}^{2}(u)\right)^{n_{j}}+\Delta \geqq 4(b-1)-\underline{\operatorname{degr}}_{w} \prod_{\mathscr{r}}\left(l_{j}^{2}\right)^{n_{j}}+\rho(H)>-4 .
$$

Because of (7-22), Lemma 7.2 is completely proved. 
All what remains to show is the following

Lemma 7.3. There is an $\varepsilon_{0}>0$ such that the following statement holds: For all $0<\varepsilon<\varepsilon_{0}$, for all $\xi, \mathscr{S} \subseteq \mathscr{V}_{\xi}$ and all $y \in Y(\xi, \mathscr{S})$ there exists a constant $c_{\xi, \mathscr{S}, y}$, so that

$$
\overline{\mathscr{J}}_{\xi, \mathscr{S}, y}^{\mathrm{int}} \leqq c_{\xi, \mathscr{S}, y} .
$$

Combining this statement with Lemma 7.2, the IR-lemma follows directly.

Proof of Lemma 7.3. At first, note that

$$
\min _{i \in I(\xi, \mathscr{S})}\left|P_{i y}\left(v^{\prime}, t_{1}^{\prime}\right) T_{i y}\left(z^{\prime}\right)\right| \leqq \min _{i \in I(\xi, \mathscr{S})}\left|T_{i y}\left(z^{\prime}\right)\right| \cdot \max _{y \in Y(\xi, \mathscr{S}), i \in I(\xi, \mathscr{P})}\left|P_{i y}\left(v^{\prime}, t_{1}^{\prime}\right)\right| .
$$

By $\left\|t_{1}^{\prime}\right\|=1,\left\|v_{i}^{\prime}\right\| \leqq 1$ for all $i=1, \ldots, r-s-1$, the inner integrals in (7-14) can be estimated by

$$
\frac{\text { const }}{\varepsilon^{L}} \cdot \min _{i \in I(\xi, \mathscr{S})}\left|T_{i y}\left(z^{\prime}\right)\right|
$$

where $L$ is a non-negative integer. Consequently, for an appropriate constant $c_{\xi, \mathscr{S}}$,

$$
\overline{\mathscr{J}}_{\xi, \mathscr{S}, y}^{\mathrm{int}}\left(t_{1}\right) \leqq c_{\xi, \mathscr{P}^{\cdot}} \int_{l_{l}^{2}\left(z^{\prime}, t_{1}^{\prime}\right) \leqq \varepsilon^{2} \text { in } \mathscr{S}} d^{4} z_{1}^{\prime} \cdots d^{4} z_{s}^{\prime} \frac{\min _{S}^{\prime \in I(\xi, \mathscr{S})}\left|T_{i y}\left(z^{\prime}\right)\right|}{\prod_{\mathscr{S}}\left(l_{j}^{2}\left(z^{\prime}, t_{1}^{\prime}\right)\right)^{n_{j}}} .
$$

It can easily be seen that for small enough $\varepsilon>0$, the integral (7-28) vanishes whenever one can find $l_{i} \in \mathscr{S}$ so that $d_{i} \neq 0$ in the representation

$$
l_{i}\left(z^{\prime}, t_{1}^{\prime}\right)=\sum_{j=1}^{s} c_{i j} z_{j}^{\prime}+d_{i} t_{1}^{\prime} .
$$

This follows from $z_{1}^{\prime}, \ldots, z_{s}^{\prime} \in \mathscr{S}$. For, the set of $z^{\prime}$ satisfying $z_{j}^{\prime 2} \leqq \varepsilon^{2}$ for all $j=1, \ldots, s$ and $l_{i}^{2}\left(z^{\prime}, t_{1}^{\prime}\right) \leqq \varepsilon^{2}$ for some $l_{i} \in \mathscr{S}$ is empty if $d_{i} \neq 0$ and $\varepsilon>0$ is small enough. More precisely, let $\left(x_{1}^{\prime}, \ldots, x_{s}^{\prime}\right)$ be a point of the integration domain of (7-28). Then, for every $l_{i} \in \mathscr{S}$

$$
\left|d_{i}\right|=\left\|d_{i} t_{1}^{\prime}\right\| \leqq\left\|l_{i}\left(x^{\prime}, t_{1}^{\prime}\right)\right\|+\sum_{j=1}^{s}\left|c_{i j}\right| \cdot\left\|x_{j}^{\prime}\right\| \leqq \varepsilon(1+s|c|)
$$

where $|c|=\max _{i, j}\left|c_{i j}\right|$, hence

$$
\varepsilon \geqq \frac{\left|d_{i}\right|}{1+s|c|} \text { for all } l_{i} \in \mathscr{S} .
$$

If there is $l_{i} \in \mathscr{S}$ with $d_{i} \neq 0$, set

$$
0<\varepsilon \leqq \frac{1}{2} \frac{\left|d_{i}\right|}{1+s|c|}
$$

Then the integration domain is empty. Consequently it is sufficient to discuss only those $\mathscr{S}$ such that $d_{i}=0$ for all $l_{i} \in \mathscr{S}$, i.e. $l_{i}=l_{i}\left(z^{\prime}\right)$ for all $l_{i} \in \mathscr{S}$. In particular, all integrals (7-28) are constant.

Finally we show that all conditions to apply the hypothesis of induction are 
satisfied by (7-28). Let

$$
z^{(1)}=l_{i_{1}}, \ldots, z^{(s)}=l_{i_{s}}
$$

be a basis of $\mathscr{S}$ with respect to $\left(z^{\prime}\right)=\left(z_{1}^{\prime}, \ldots, z_{s}^{\prime}\right)$ and let $H_{1}, \ldots, H_{t}$ be a sequence of classes of affine subspaces of $\left(z^{\prime}\right)$ which is ordered with respect to this basis, i.e.

a. $H_{j}$ is parametrized by $\left(\tilde{k}_{j}\right) \subseteq\left\{z^{(1)}, \ldots, z^{(s)}\right\} \subseteq \mathscr{S}$ for $j=1, \ldots, t$.

b. The $\left(\tilde{k}_{j}\right)$ contain the $\left(\tilde{k}_{h}\right)$ for $j>h$.

Every basis (7-32) can be completed with $w=l_{\xi}$ and $v_{1}, \ldots, v_{r-s-1}$ of (7-8a) to a basis

$$
l_{i_{1}}, \ldots, l_{i_{s}}, \quad w, v_{1}, \ldots, v_{r-s-1}
$$

of $\mathscr{W}$ with respect to $(u)=\left(u_{1}, \ldots, u_{r}\right), u=f_{\xi \mathscr{S}}\left(z^{\prime}, v, w\right)$ (cp. (7-8b)). To every $H_{j}$ in (7-33) we associate in this way an affine subspace of $(u)$ which is parametrized by $\left(\widetilde{k}_{j}\right)$, and we associate a corresponding $\rho\left(H_{j}\right) \in \mathbf{Z}$. By construction of $I(\xi, \mathscr{S})$ and by

$$
\underline{\operatorname{degr}} \tilde{k}_{j} T_{i y}\left(z^{\prime}\right) \geqq \underline{\operatorname{degr}_{\tilde{k}_{j}}} M_{i}\left(u\left(z^{\prime}, v, w\right)\right) ; \quad \text { for all } j=1, \ldots, t \text { and all } i \in I
$$

(Lemma 7.2), there is $i \in I(\xi, \mathscr{S})$, so that

$$
\operatorname{\operatorname {degr}}_{\tilde{k}_{j}} T_{i y}\left(z^{\prime}\right) \geqq \underline{\operatorname{degr}}{\underline{\tilde{k}_{j}}} M_{i}(u) \geqq \rho\left(H_{j}\right) \quad \text { for all } \quad j=1, \ldots, t .
$$

Hence the given IR-set, restricted to subspaces $H$ of the above form, is also one with respect to the numerator of (7-28).

Let

$$
x_{1}=l_{i_{1}}, \ldots, x_{p}=l_{i_{p}}, \quad y_{1}=l_{k_{1}}, \ldots, y_{s-p}=l_{k_{s-p}}
$$

be an arbitrary basis of $\mathscr{S}$ with respect to $\left(z_{1}^{\prime}, \ldots, z_{s}^{\prime}\right)$, so that $z^{\prime}=z^{\prime}(x, y)$. Let $H$ be the affine subspace of $\left(z_{1}^{\prime}, \ldots, z_{s}^{\prime}\right)$ which is parametrized by $x_{1}, \ldots, x_{p}$, and $y_{1}, \ldots, y_{s-p}$ are held fixed. Then

$$
\begin{aligned}
& 4 p+\rho(H)-\underline{\operatorname{degr}} x \prod_{\mathscr{S}}\left(l_{j}^{2}\left(z^{\prime}(x, y)\right)\right)^{n_{J}} \\
& \geqq 4 p+\rho(H)-\underline{\operatorname{degr}_{x}} \prod_{\mathscr{V}}\left(l_{j}^{2}\left(u\left(z^{\prime}, v, w\right)\right)\right)^{n_{J}} \\
& >0
\end{aligned}
$$

by assumption. Hence the hypothesis of induction applies to the integrals (7-28), and consequently they are convergent. This completes the proof of Lemma 7.3 and of the IR-lemma.

\section{Proof of the Auxiliary Theorem}

The idea of proof is rather simple. The integral (4-6) is divided into a sum of integrations over appropriate sections. In every sector the numerator is estimated by one argument of the outer minimum of (4-8). The resulting integrals are of a form which allows application of Lemma 7.1 and the auxiliary power counting theorem of [1], giving the desired cutoff dependence.

At first, $\overline{\mathscr{I}}_{\lambda}$ is written as

$$
\overline{\mathscr{I}}_{\lambda}=\sum_{\mathscr{S} \subseteq \mathscr{S}_{0}} \overline{\mathscr{I}}_{\lambda \mathscr{S}}
$$


where the sum goes over all subsets $\mathscr{S} \subseteq \mathscr{S}_{0}=\left\{l_{i} \in \mathcal{N} \mid \mu_{i}=0\right\}$, and

$$
\overline{\mathscr{I}}_{\lambda, \mathscr{S}}(q, \mu)=\int_{\substack{l_{l}^{2} \leqq \varepsilon^{2} \ln \mathscr{S} \\ l_{l}^{2} \geqq \varepsilon^{2} \ln \mathscr{T}}}^{\mu, \mathscr{L}} d^{4} k_{1} \cdots d^{4} k_{m} \frac{Z(\lambda, k, q)}{\prod_{\mathscr{N}}\left(l_{j}^{2}+\mu_{j}^{2}\right)^{n_{j}}},
$$

where we have written $\mathscr{T}=\mathscr{S}_{0} \backslash \mathscr{S}$, and $\varepsilon>0$ is a constant. The integration domain is restricted to those $k$ satisfying $l_{i}^{2}(k, q) \leqq \varepsilon^{2}$ for all $l_{i} \in \mathscr{S}$ and $l_{i}^{2}(k, q) \geqq \varepsilon^{2}$ for all $l_{i} \in \mathscr{T}$. For every $\mathscr{S}$ choose a basis

$$
u_{1}=l_{i_{1}}, \ldots, u_{r}=l_{i_{r}}
$$

of $\mathscr{S}$ with respect to $k$ and complete it by

$$
v_{1}=l_{j_{1}}, \ldots, v_{m-r}=l_{J_{m-r}}
$$

to a basis of $\mathscr{L}$ with respect to $k$. We write $l_{i}=l_{i}(u, v, q)$. Every $l_{i} \in \mathscr{S}$ has a representation

$$
l_{i}=U_{i}+\bar{Q}_{i}(q), \quad U_{i}=\sum_{j=1}^{r} \hat{C}_{i j} u_{j} .
$$

Without loss of generality let the Jacobian for $k \rightarrow u, v$ be equal to one. Then

$$
\overline{\mathscr{I}}_{i, \mathscr{S}}(q, \mu) \leqq \int_{l_{i}^{2} \leqq \varepsilon^{2} \text { in } \mathscr{S}} d^{4} u_{1} \cdots d^{4} u_{r} \int_{l_{l}^{2} \geq \varepsilon^{2} \text { in } \mathscr{T}}^{i, \mathscr{P} \mathscr{\mathscr { S }}} d^{4} v_{1} \cdots d^{4} v_{m-r} \frac{Z(\lambda, k(u, v, q), q)}{\prod_{\mathcal{N}}\left(l_{j}^{2}+\mu_{j}^{2}\right)^{n_{j}}} .
$$

As in Sect. 7 it can easily be seen that there is $\varepsilon_{0}=\varepsilon_{0}(\bar{Q}, \hat{C}, r)>0$, so that for $0<\varepsilon<\varepsilon_{0}, \overline{\mathscr{I}}_{2, \mathscr{S}}(q, \mu)=0$ if $\bar{Q}_{j} \neq 0$ for some $l_{j} \in \mathscr{S}$. In the following we assume that $\bar{Q}_{j}=0$ whenever $l_{j} \in \mathscr{S}$, so that $l_{j}=l_{j}(u)$.

By assumption, the numerator $Z(\lambda, k, q)$ is admissible with respect to the given degree set. For any $\mathscr{S}$ we take the $i \in I$ of (4-8) which corresponds to $\mathscr{S}$ by Definition 4.2. Then

$$
\begin{aligned}
& \overline{\mathscr{I}}_{\lambda \mathscr{S}}(q, \mu) \leqq \int_{l_{\iota}^{2} \leqq \delta^{2} \ln \mathscr{S}} d^{4} u_{1} \cdots d^{4} u_{r} \frac{\min _{j \in J_{l}}\left|M_{i j}(u, q)\right|}{\prod_{\mathscr{S}}\left(l_{j}^{2}(u)\right)^{n_{J}}} \\
& \cdot \int_{l_{i}^{2} \geqq \varepsilon^{2} \ln \mathscr{T}}^{i, \mathscr{L} \mathscr{\mathscr { T }}} d^{4} v_{1} \cdots d^{4} v_{m-r} \frac{\min _{l \in K_{i}} \lambda^{-p_{u l}}\left|C_{i l}(k(u, v, q), q)\right|}{\left(\prod_{\mathscr{T}}\left(l_{j}^{2}(u, v, q)\right)^{n_{j}}\right)\left(\prod_{\mathscr{N} \backslash \mathscr{S}_{0}}\left(l_{j}^{2}(u, v, q)+\mu_{j}^{2}\right)^{n_{j}}\right)} .
\end{aligned}
$$

To the inner integral we now apply the auxiliary power counting theorem of [1], while the outer integral will be estimated by the IR-lemma.

Lemma 8.1. Set

$$
\overline{\mathscr{I}}_{i, \mathscr{S}}^{e}(q, u, \mu)=\int_{l_{i}^{2} \geq c^{2} \text { in } \mathscr{T}}^{\mu, \mathscr{L} \backslash \mathscr{S}} d^{4} v_{1} \cdots d^{4} v_{m-r} \frac{\min _{l \in K_{l}} \lambda^{-p_{i l}}\left|C_{i l}(k(u, v, q), q)\right|}{\prod_{\mathscr{T}}\left(l_{j}^{2}(u, v, q)\right)^{n_{j}} \prod_{\mathcal{N} \backslash \mathscr{S}_{0}}\left(l_{j}^{2}(u, v, q)+\mu_{j}^{2}\right)^{n_{j}} .}
$$


There exist $K_{\mathscr{S}}(\mu, q)>0$ and $c_{\mathscr{S}}(\mu, q)>0$, so that for all $\left(u_{1}, \ldots, u_{r}\right) \in \mathbf{R}^{4 r}$, satisfying $l_{i}^{2}(u) \leqq \varepsilon^{2}$ for all $l_{i} \in \mathscr{S}$, we have

$$
\begin{aligned}
& \overline{\mathscr{I}}_{\lambda \mathscr{S}}^{e}(q, u, \mu) \leqq c_{\mathscr{S}}(\mu, q) \\
& \cdot\left\{\begin{array}{ll}
1 & \text { if } \max _{H \in \mathscr{H}^{\mathrm{Uv}}} \omega(H)<0 \\
\lambda^{-1} \log ^{m} \lambda & \text { if } \max _{H \in \mathscr{H}^{\mathrm{Uv}}} \omega(H)<0 \\
\lambda^{\max _{H \in \mathscr{H}} \mathrm{Uv}[\omega(H)]} \log ^{m} \lambda & \text { if } \max _{H \in \mathscr{H}} \mathrm{Uv} \omega(H) \geqq 0
\end{array} \text { and if all } p_{i l} \geqq 1\right.
\end{aligned}
$$

for all $\lambda>K_{\mathscr{S}}(\mu, q)$, where the ultraviolet divergence degrees $\omega(H)$ are given by (4-17).

Proof. Let $\mathscr{R}$ be the set of all $l_{i} \in \mathscr{N} \backslash \mathscr{S}$ which depend only on $u$ and $q$. Then

and

$$
\frac{1}{\left[l_{j}^{2}(u, q)\right]^{n_{j}}} \leqq \frac{1}{\varepsilon^{2 n_{j}}} \quad \text { for } \quad l_{j} \in \mathscr{T} \cap \mathscr{R}
$$

$$
\frac{1}{\left[l_{j}^{2}(u, q)+\mu_{j}^{2}\right]^{n_{j}}} \leqq \frac{1}{\left(\mu_{j}^{2}\right)^{n_{j}}} \quad \text { for } \quad l_{j} \in\left(\mathscr{N} \backslash \mathscr{S}_{0}\right) \cap \mathscr{R} \quad\left(\mu_{j}^{2}>0 !\right) .
$$

Hence without loss of generality we assume $\mathscr{R}=\emptyset$. For $l_{j} \in \mathscr{T}$ we have

$$
\frac{l_{j}^{2}+\eta^{2}}{l_{j}^{2}} \leqq 1+\frac{\eta^{2}}{\varepsilon^{2}}
$$

for any $\eta^{2}>0$. Consequently

$$
\begin{aligned}
\overline{\mathscr{I}}_{\lambda \mathscr{S}}^{e}(q, u, \mu) & \leqq c \int_{l_{1}^{2} \geqq \varepsilon^{2} \text { in } \mathscr{T}}^{\lambda, \mathscr{L} \backslash \mathscr{S}} d^{4} v_{1} \cdots d^{4} v_{m-r} \frac{\min _{l \in K_{i}} \lambda^{-p_{i l}}\left|C_{i l}(k(u, v, q), q)\right|}{\prod_{\mathscr{T}}\left(l_{j}^{2}+\eta^{2}\right)^{n_{j}} \cdot \prod_{\mathscr{N} \backslash \mathscr{S}_{0}}\left(l_{j}^{2}+\mu_{j}^{2}\right)^{n_{j}}} \\
& \leqq c \int_{\min _{l \in K_{i}} \lambda^{-p_{i l}}\left|C_{i l}(k(u, v, q), q)\right|}^{\lambda, \mathscr{L} \backslash \mathscr{S}} d^{4} v_{1} \cdots d^{4} v_{m-r} \frac{\prod_{\mathscr{T}}\left(l_{j}^{2}(u, v, q)+\eta^{2}\right)^{n_{j}} \cdot \prod_{\mathcal{N} \backslash \mathscr{S}_{0}}\left(l_{j}^{2}(u, v, q)+\mu_{j}^{2}\right)^{n_{j}}}{(8-12)}
\end{aligned}
$$

where $c=c(\varepsilon)$ is a constant. Now let

$$
w_{1}=l_{i_{1}}, \ldots, w_{d}=l_{i_{d}}, \quad z_{1}=l_{l_{d+1}}, \ldots, z_{m-r-d}=l_{l_{m-r}}
$$

be an arbitrary basis of $\mathscr{L} \backslash \mathscr{S}$ with respect to $\left(v_{1}, \ldots, v_{m-r}\right)$. Variable $w$ and constant $z$ define a class $H$ of affine subspaces of $\left(v_{1}, \ldots, v_{m-r}\right)$. The set of all such $H$, for all bases (8-13), is denoted by $\mathscr{H}_{\mathscr{S}}^{\mathrm{UV}}$. Every basis $(8-13)$ of $\mathscr{L} \backslash \mathscr{S}$ can be completed to a basis of $\mathscr{L}$ with respect to $k$ by adding $u_{1}, \ldots, u_{r}$ of (8-3a). In this way, every $H \in \mathscr{H}_{\mathscr{S}}^{\mathrm{UV}}$ is considered as a subspace of $(k)$, where $(z)$ and $(u)$ are held fixed. This means $\mathscr{H}_{\mathscr{S}}^{\mathrm{UV}} \subseteq \mathscr{H}^{\mathrm{UV}}$. To every $H \in \mathscr{H}_{\mathscr{S}}^{\mathrm{UV}}$ we associate the corresponding $\delta(H, \mathscr{S})$ of the given degree set.

Every sequence $H_{1}, \ldots, H_{s} \in \mathscr{H}_{\mathscr{S}}^{\mathrm{UV}}$ which is ordered with respect to the basis (8-13) of $\mathscr{L} \backslash \mathscr{S}$ is a sequence of subspaces which is ordered in $(w, z)$ with respect to the basis $(w, z, u)$ of $\mathscr{L}$. Hence, by assumption, for every such sequence there exists $l \in K_{i}$ so that

$$
\overline{\operatorname{degr}}_{x_{g} \mid y_{g}} C_{i l}-p_{i l} \leqq \overline{\operatorname{degr}}_{x_{g} \mid y_{g} u_{1} \cdot u_{r}} C_{i l}-p_{i l} \leqq \delta\left(H_{g}, \mathscr{S}\right) \text { for all } g=1, \ldots, s
$$


where $\left(x_{g}\right)$ are the parameters and $\left(y_{g}\right)$ are the complementary parameters of $H_{g}$ with respect to (8-13), i.e. $\left(x_{g}, y_{g}\right)=(w, z)$. This means that the set $\left\{\delta(H, \mathscr{T}) \mid H \in \mathscr{H}_{\mathscr{S}}^{\mathrm{UV}}\right\}$ is a UV-set for the numerator of (8-12) in the sense of [1] which is independent of $u$. Furthermore, for any $H \in \mathscr{H}_{\mathscr{S}}^{\mathrm{UV}}$, parametrized by $(x)=\left(x_{1}, \ldots, x_{e}\right)$, we have

$$
\begin{aligned}
\omega_{\mathscr{S}}(H) & \equiv 4 e+\delta(H, \mathscr{S})-\overline{\operatorname{degr}}_{x} \prod_{\mathscr{T}}\left(l_{j}^{2}+\mu_{j}^{2}\right)^{n_{j}}-\overline{\operatorname{degr}}_{x} \prod_{\mathscr{N} \mathscr{P}_{0}}\left(l_{j}^{2}+\mu_{j}^{2}\right)^{n_{J}} \\
& \leqq \omega(H),
\end{aligned}
$$

because of $\delta(H, \mathscr{S}) \leqq \delta(H)$, where $\omega(H)$ and $\delta(H)$ are given by (4-17) and (4-18), respectively. Thus, all the conditions are met to apply the power counting theorem of [1] to (8-12). Hence, there exist $K_{\mathscr{S}}(\mu, q)>0$ and $c_{\mathscr{S}}(\mu, q)>0^{6}$, so that for all $\lambda>K_{\mathscr{S}}(\mu, q)$

$$
\begin{aligned}
& \overline{\mathscr{I}}_{\lambda \mathscr{S}}^{e}(q, u, \mu) \leqq c_{\mathscr{S}}(\mu, q)
\end{aligned}
$$

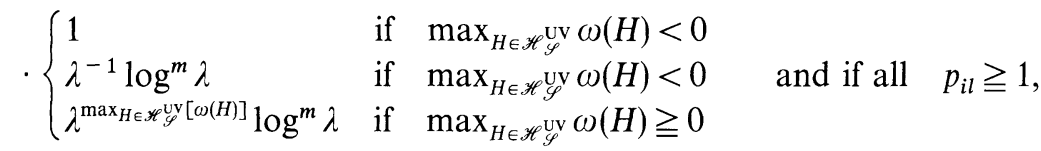

$$
\begin{aligned}
& \leqq c_{\mathscr{Y}}(\mu, q) \\
& \cdot\left\{\begin{array}{ll}
1 & \text { if } \max _{H \in \mathscr{H}^{\mathrm{Uv}}} \omega(H)<0 \\
\lambda^{-1} \log ^{m} \lambda & \text { if } \max _{H \in \mathscr{H}} \mathrm{Uv} \omega(H)<0 \\
\lambda^{\max _{H \in \mathscr{H}} \mathrm{vV}[\omega(H)]} \log ^{m} \lambda & \text { if } \max _{H \in \mathscr{H}} \mathrm{Uv} \omega(H) \geqq 0
\end{array} \text { and if all } p_{i l} \geqq 1 .\right.
\end{aligned}
$$

Having determined the cutoff dependence of the inner integrals, we now turn to the remaining integrations.

Lemma 8.2. The integral

$$
\overline{\mathscr{I}}_{\mathscr{S}}(q)=\int_{l_{i}^{2} \leq \varepsilon^{2} \operatorname{In} \mathscr{\mathscr { S }}} d^{4} u_{1} \cdots d^{4} u_{r} \frac{\min _{j \in J_{i}}\left|M_{i j}(u, q)\right|}{\prod_{\mathscr{S}}\left(l_{j}^{2}(u)\right)^{n_{j}}}
$$

is convergent for every $\mathscr{S}$.

Combined with Lemma 8.1 this means that there are $K(\mu, q)>0$ and $c(\mu, q)>0$, so that

$$
\begin{aligned}
& \overline{\mathscr{I}}_{\lambda}(q, \mu) \leqq c(\mu, q) \\
& \cdot\left\{\begin{array}{ll}
1 & \text { if } \max _{H \in \mathscr{H}} \mathrm{Uv} \omega(H)<0 \\
\lambda^{-1} \log ^{m} \lambda & \text { if } \max _{H \in \mathscr{H}} \mathrm{Uv} \omega(H)<0 \\
\lambda^{\max } \lambda \in \mathscr{H}^{\mathrm{Uv}[\omega(H)]} \log ^{m} \lambda & \text { if } \max _{H \in \mathscr{H}} \mathrm{Uv} \omega(H) \geqq 0
\end{array} \text { and all } \quad p_{i l} \geqq 1\right.
\end{aligned}
$$

for all $\lambda>K(\mu, q)$, which completes the proof of the auxiliary theorem.

${ }^{6} u$ is bounded and $\{\delta(H, \mathscr{S})\}$ is independent of $u$, hence $K_{\mathscr{S}}$ and $c_{\mathscr{S}}$ can be chosen to be independent of $u$ 
Proof of Lemma 8.2. We use the IR-lemma of Sect. 7. Every integral (8-18) is of the form (7-1), where $\mathscr{S}$ stand for $\mathscr{W}=\mathscr{V}$ and $\overline{\mathscr{I}}_{\mathscr{S}}$ for $\overline{\mathscr{J}}$.

Let

$$
z_{1}=l_{i_{1}}, \ldots, z_{s}=l_{i_{s}}, \quad w_{1}=l_{i_{s+1}}, \ldots, w_{r-s}=l_{i_{r}}
$$

be a basis of $\mathscr{S}$ with respect to $\left(u_{1}, \ldots, u_{r}\right)$. We define $\mathscr{H}_{\mathscr{S}}^{\mathrm{IR}}$ as the set of all classes $H$ of affine subspaces of $\left(u_{1}, \ldots, u_{r}\right)$ which are given by constant $w_{1}, \ldots, w_{r-s}$, for arbitrary bases (8-20). Every such basis of $\mathscr{S}$ can be completed with $v_{1}, \ldots, v_{m-r} \in \mathscr{L} \backslash \mathscr{S}$ of $(8-3 \mathrm{~b})$ to a basis of $\mathscr{L}$ with respect to $k$. In this way, every $H \in \mathscr{H}_{\mathscr{S}}^{\mathrm{IR}}$ can be identified with a subspace of $(k)$ and we can associate to $H$ the corresponding $\rho(H, \mathscr{S})$.

Every sequence $H_{1}, \ldots, H_{t} \in \mathscr{H}_{\mathscr{g}}^{\mathrm{IR}}$ which is ordered with respect to the basis $(8-20)$ of $\mathscr{S}$ is a sequence of subspaces which is ordered in $(z, w)$ with respect to the basis $(z, w, v)$ of $\mathscr{L}$. By assumption, there is a $j \in J_{i}$, so that

$$
\underline{\operatorname{degr}}_{x_{g} \mid y_{g}} M_{i j} \geqq \underline{\operatorname{degr}}_{x_{g} \mid y_{g} v_{1} \cdots v_{m-r}} M_{i j} \geqq \rho\left(H_{g}, \mathscr{S}\right) \text { for all } g=1, \ldots, t,
$$

where $\left(x_{g}\right)$ are the parameters and $\left(y_{g}\right)$ are the complementary parameters of $H_{g}$. Hence, $\left\{\rho(H, \mathscr{S}) \mid H \in \mathscr{H}_{\mathscr{S}}^{\mathrm{IR}}\right\}$ is an IR-set for the numerator of $(8-18)$ in the sense of Definition 7.1. For every $H \in \mathscr{H}_{\mathscr{S}}^{\mathrm{IR}}$, parametrized by $(z)=\left(z_{1}, \ldots, z_{s}\right)$,

$$
\begin{aligned}
r_{\mathscr{S}}(H) & \equiv 4 s+\rho(H, \mathscr{S})-\underline{\operatorname{degr}_{z}} \prod_{\mathscr{S}}\left[l_{j}^{2}(u(z, v))\right]^{n_{J}} \\
& \geqq 4 s+\rho(H)-\underline{\operatorname{degr}_{z}} \prod_{\mathscr{N}}\left(l_{j}^{2}\right)^{n_{j}} \\
& >0
\end{aligned}
$$

by assumption of the auxiliary theorem, where $\rho(H)$ is given by (4-21). Consequently, all conditions to apply the IR-lemma are satisfied, and Lemma 8.2 is proved.

\section{Conclusions}

We have generalized the convergence theorem for Feynman integrals with a lattice cutoff of [1] to lattice field theories with massless fields. Infrared power counting conditions are sufficient for the convergence of diagrams with finite lattice cutoff. If these conditions are supplemented by the ultraviolet power counting conditions of [1], the continuum limit of a lattice Feynman integral exists and is equal to the formal limit, i.e. the integral over the continuum limit of the integrand. Apart from the possibility of zero-mass propagators, the general assumptions on the structure of the lattice integrand are the same as in the massive case [1]. It should be periodic with the Brillouin zone in every loop momentum, the propagators should have only one pole in the Brillouin zone, and the line momenta should be natural. While the last condition can always be satisfied by an appropriate choice of the loop momenta, the pole condition is a genuine restriction. In particular, the power counting theorem does not apply to fermions with propagators having poles on the boundary of the Brillouin zone. Such propagators would require stronger assumptions to be made on the structure of Feynman integrands on the lattice, in addition to the periodicity. 
In a forthcoming paper, following the ideas of Lowenstein and Zimmermann, the power counting theorem will be used to construct a renormalization scheme for a wide class of lattice field theories containing massless fields [6].

\section{Appendix A. UV- and IR-Degrees for Polynomials}

Let $P$ be a polynomial in variables $u, w$ and $q . P$ can be written as

$$
P(u, w, q)=\sum_{\alpha} Q_{\alpha}(w, q) \cdot M_{\alpha}(u),
$$

where $M_{\alpha}$ are linearly independent homogeneous polynomials and

$$
Q_{\alpha}(w, q) \neq \equiv 0 \text { in } w \text { ( } q \text { fixed!). }
$$

The UV-degree of $P$ with respect to $u$ is defined by

$$
\overline{\operatorname{degr}}_{u \mid w} P \equiv \max _{\alpha} \operatorname{degr} M_{\alpha},
$$

and the IR-degree is defined by

$$
\underline{\operatorname{degr}}_{u \mid w} P \equiv \min _{\alpha} \operatorname{degr} M_{\alpha},
$$

where degr $M_{\alpha}$ is the homogeneity degree of $M_{\alpha}$. Note that the degrees defined in this way depend on the external momenta $q$. Sometimes for the UV-degree we will use the shorthand notation

In general,

$$
\overline{\operatorname{degr}}_{u} P(u, w, q) \equiv \overline{\operatorname{degr}}_{u \mid w q} P(u, w, q) .
$$

$$
\overline{\operatorname{degr}}_{u \mid w} P(u, w, q) \leqq \overline{\operatorname{degr}}_{u \mid w q} P(u, w, q)
$$

and

$$
\underline{\operatorname{degr}}_{u \mid w} P(u, w, q) \geqq \underline{\operatorname{deg}}_{u \mid w q} P(u, w, q) .
$$

For "exceptional" momenta $q$, the latter is a strict inequality. If $P$ is the denominator of a momentum space Feynman integrand, these momenta destroy the convergence of the Feynman integral, hence they must be excluded.

We list the most important properties of $\overline{\operatorname{degr}}$ and degr. Let $F, F_{1}, \ldots, F_{r}$ be polynomials in $u, w, q$. Then

$$
\begin{aligned}
\overline{\operatorname{degr}}_{u \mid w} F^{n} & =n \overline{\operatorname{degr}}_{u \mid w} F, \\
\overline{\operatorname{degr}}_{u \mid w} F^{n} & =n \overline{\operatorname{degr}}_{u \mid w} F, \\
\overline{\operatorname{degr}}_{u \mid w} \prod_{j=1}^{r} F_{j} & =\sum_{j=1}^{r} \overline{\operatorname{degr}}_{u \mid w} F_{j}, \\
\overline{\operatorname{degr}}_{u \mid w} \prod_{j=1}^{r} F_{j} & =\sum_{j=1}^{r} \overline{\operatorname{degr}}_{u \mid w} F_{j}, \\
\overline{\operatorname{degr}}_{u \mid w} \sum_{j=1}^{r} F_{j} & \leqq \\
\max & \overline{\operatorname{degr}}_{u \mid w} F_{j}, \ldots, r
\end{aligned}
$$




$$
\underline{\operatorname{degr}}_{u \mid w} \sum_{j=1}^{r} F_{j} \geqq \min _{j=1, \ldots, r} \underline{\operatorname{degr}}_{u \mid w} F_{j} .
$$

At two stages of this paper we need the following

Lemma A.1. Let $P$ be a polynomial in variables $u, v . P$ can be written as

$$
P(u, v)=\sum_{\alpha} R_{\alpha}(u) Q_{\alpha}(v), \quad Q_{\alpha} \neq 0,
$$

where $R_{\alpha}$ are linearly independent homogeneous polynomials, so that all polynomials $Q_{\alpha}$ for $\alpha$ with the same $\operatorname{degr} R_{\alpha}$ are linearly independent.

Let $u=f(\tilde{u}, \hat{u})$ be linear and homogeneous. Then

$$
\operatorname{degr}_{\tilde{u} \mid u} R_{\alpha}(u) \geqq \underline{\operatorname{deg}} \underline{\tilde{u}}_{\tilde{u} \mid u v} P(u, v) \quad \text { for all } \alpha .
$$

Proof. $P$ can always be decomposed into linearly independent homogeneous polynomials $M_{\alpha}(u)$ :

For any $\beta$ let

$$
P(u, v)=\sum_{\alpha} M_{\alpha}(u) Q_{\alpha}(v), \quad Q_{\alpha}(v) \neq \equiv .
$$

with $n$ maximal and

$$
\beta=\operatorname{degr} M_{\alpha_{1}}=\cdots=\operatorname{degr} M_{\alpha_{n}}
$$

$$
Q_{\alpha_{1}}, \ldots, Q_{\alpha_{t}}, \quad t \leqq n
$$

be linearly independent with $t$ maximal, so that

Then

$$
Q_{\alpha_{i}}=\sum_{j=1}^{t} c_{i j} Q_{\alpha_{j}} \text { for all } i \text { with } t<i \leqq n
$$

where

$$
\sum_{j=1}^{n} M_{\alpha_{j}}(u) Q_{\alpha_{j}}(v)=\sum_{i=1}^{t} R_{\alpha_{i}}(u) Q_{\alpha_{i}}(v)
$$

$$
R_{\alpha_{i}}(u)=M_{\alpha_{i}}(u)+\sum_{j=t+1}^{n} c_{j i} M_{\alpha_{j}} .
$$

The $R_{\alpha_{i}}, i=1, \ldots, t$ are linearly independent and homogeneous of degree $\beta$. Doing so for all $\beta$, the first part of the lemma follows.

Let $u=f(\tilde{u}, \hat{u})$ be linear and homogeneous. Write every $R_{\alpha}$ in a partition (A-9) as

$$
R_{\alpha}(u)=\sum_{\beta} S_{\beta}(\tilde{u}) V_{\alpha \beta}(\hat{u}), \quad V_{\alpha \beta}(\hat{u}) \not \equiv 0,
$$

where $S_{\beta}$ are linearly independent homogeneous polynomials in $\tilde{u}$. Every $V_{\alpha \beta}(\hat{u})$ is homogeneous in $\hat{u}$ of degree $\operatorname{degr} R_{\alpha}-\operatorname{degr} S_{\beta}$. Inserting this in (A-9) yields

$$
P(u, v)=\sum_{\beta} S_{\beta}(\tilde{u}) \sum_{\alpha} Q_{\alpha}(v) V_{\alpha \beta}(\hat{u}) .
$$

The first sum is over all $\beta$ for which $\alpha$ exists with $V_{\alpha \beta}(\hat{u}) \neq \equiv$. For every $\beta$

$$
\sum_{\alpha} Q_{\alpha}(v) V_{\alpha \beta}(\hat{u}) \neq 0
$$

because of the linear independence of the $Q_{\alpha}$ for $\alpha$ having the same $\operatorname{degr} R_{\alpha}$. Hence 


$$
\operatorname{degr}_{\tilde{u} \mid \hat{u}} R_{\alpha}(u) \geqq \underline{\operatorname{degr}} \underline{\tilde{u}} \tilde{u} \mid \hat{v}_{P} P(u, v) \quad \text { for all } \alpha .
$$

\section{Appendix B. Proof of the Corollary to the Auxiliary Theorem}

To prove the corollary of Sect. 4, the integral $\overline{\mathscr{J}}_{\lambda},(4-24)$, will be estimated by a finite sum of integrals of the form (4-6) to which the auxiliary theorem applies, and such that the numerators of the integrands are admissible with respect to the degree set $\mathscr{E}$, consisting of all $\delta(H, \mathscr{S})=\operatorname{degr}_{v \mid z} P, H \in \mathscr{H}{ }^{\mathrm{uv}}$, and of all $\rho(H, \mathscr{S})=$ $\underline{\operatorname{degr}}_{u \mid w} P, H \in \mathscr{H}^{\mathrm{IR}}$ (cp. (4-24)f). Note that the $\rho$ and $\delta$ are independent of $\mathscr{S} \subseteq \mathscr{S}_{0}$.

We first mention the following fact. Let $P(u, v, q)$ be a polynomial and

$$
P(u, v, q)=\sum_{g} R_{g}(u) Q_{g}(v, q) ; \quad Q_{g}(v, q) \neq 0 \quad \text { in } v,
$$

a decomposition of $P$ into linearly independent homogeneous polynomials $R_{g}$ in $u$. Let

$$
u^{\prime}=D u+E q, \quad v^{\prime}=A v+B u+C q
$$

be an arbitrary linear transformation, where $A$ and $D$ are invertible matrices. Then, for every partition $\left(v^{\prime}\right)=\left(v^{(1)}, v^{(2)}\right)$, we get

$$
\overline{\operatorname{degr}}_{v^{(1)} \mid v^{(2)} u^{\prime}} Q_{g} \leqq \overline{\operatorname{degr}}_{v^{(1)} \mid v^{(2)} u^{\prime}} P \text { for all } g \text {. }
$$

Using the linear independence of the $R_{g}$, this follows directly from

$$
\begin{aligned}
\overline{\operatorname{degr}}_{v^{\prime} \mid v^{(2)} u^{\prime}} Q_{g}(v, q) & =\overline{\operatorname{degr}}_{v^{(1) \mid} v^{(2)} u^{\prime}} R_{g}(u) Q_{g}(v, q) \\
& \leqq \overline{\operatorname{degr}}_{v^{(1)} \mid v^{(2)} u^{\prime}} P(u, v, q) .
\end{aligned}
$$

To prove the corollary, let first $\mathscr{S} \subseteq \mathscr{S}_{0}$ be an arbitrary subset (cf. (4-9)). Let

$$
u_{1}, \ldots, u_{r}, \quad v_{1}, \ldots, v_{m-r}
$$

be a basis of $\mathscr{L}$ with respect to $k$ such that $u_{1}, \ldots, u_{r}$ is a basis of $\mathscr{S}$ with respect to $k$. Then there exists a decomposition of the numerator $P$ of (4-24) into linearly independent homogeneous polynomials $R_{g}$,

$$
P(k(u, v, q), q)=\sum_{g} R_{g}(u) Q_{g}(v, q) \quad \text { with } \quad Q_{g}(v, q) \neq 0 \quad \text { in } v,
$$

so that for every partition $(u)=\left(u^{(1)}, u^{(2)}\right)$

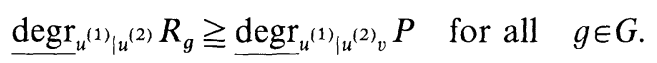

This is proved in Appendix A. A decomposition (B-5) is possible for every basis (B-4) of $\mathscr{L}$ with respect to $k$, for fixed $\mathscr{S} \subseteq \mathscr{S}_{0}$. Hence, with an appropriate set $J$,

$$
|P(k, q)| \leqq \min _{j \in J} \sum_{g \in G_{j}}\left|R_{g}(k, q)\right| \cdot\left|Q_{g}(k, q)\right| \cdot
$$

Writing $G=\otimes_{j \in J} G_{j}{ }^{7}$ and setting for $\left(g_{j}\right)_{j \in J} \in G: R_{j g}=R_{g_{J}}, Q_{j g}=Q_{g_{j}}$, we get

\footnotetext{
7 This notation is explained in Appendix B of refs. [1]
} 


$$
\begin{aligned}
|P(k, q)| & \leqq \sum_{g \in G} \min _{j \in J}\left(\left|R_{j g}(k, q)\right| \cdot\left|Q_{j g}(k, q)\right|\right) \\
& \leqq \sum_{g \in G}\left(\min _{j \in J}\left|R_{j g}(k, q)\right|\right) \sum_{j \in J}\left|Q_{j g}(k, q)\right| \\
& \leqq \sum_{l \in X}\left(\min _{j \in J}\left|M_{j l}(k, q)\right|\right) \cdot\left|C_{l}(k, q)\right|,
\end{aligned}
$$

where $X=G \otimes J$ and for $l=(g, h) \in X$ we have written $M_{j l}=R_{j g}$ and $C_{l}=Q_{h g}$.

Let

$$
u_{1}^{\prime}, \ldots, u_{r}^{\prime}, \quad v_{1}^{\prime}, \ldots, v_{m-r}^{\prime}
$$

be another basis of $\mathscr{L}$ with respect to $k$ such that $u_{1}^{\prime}, \ldots, u_{r}^{\prime}$ is a basis of $\mathscr{S}$. Such a basis is related to (B-4) by a transformation of the form (B-2). Writing $\left(v^{\prime}\right)=\left(v^{(1)}, v^{(2)}\right)$ and using (B-3), we have for all $C_{l}$ of (B-7),

$$
\overline{\operatorname{degr}}_{v^{(1)} \mid v^{(2)} u^{\prime}} C_{l} \leqq \overline{\operatorname{degr}}_{v^{(1)} \mid v^{(2)} u^{\prime}} P \text { for all } l \in X \text {. }
$$

Furthermore, by construction, all $M_{j l}$ of (B-7) depend on $u^{\prime}$ and $q$ only, and one can always find a $j \in J$, so that for each partition $\left(u^{\prime}\right)=\left(u^{(1)}, u^{(2)}\right)$,

$$
\underline{\operatorname{degr}}_{u^{(1)} \mid u^{(2)} v} M_{j l} \geqq \underline{\operatorname{degr}}_{u^{(1)} \mid u^{(2)} v} P \quad \text { for all } l \in X .
$$

Until now, $\mathscr{S}$ is held fixed. Taking the minimum of (B-7) over all $\mathscr{S} \subseteq \mathscr{S}_{0}$, we get

$$
|P(k, q)| \leqq \min _{i \in I} \sum_{l \in X_{i}}\left(\min _{j \in J_{l}}\left|M_{j l}(k, q)\right|\right) \cdot\left|C_{l}(k, q)\right| \leqq \sum_{l \in Y} Z_{l}(k, q),
$$

where

$$
Z_{l}(k, q)=\min _{i \in I}\left(\left|C_{i l}(k, q)\right| \cdot \min _{j \in J_{i}}\left|M_{i j l}(k, q)\right|\right) .
$$

$I$ is an appropriate finite set, $Y=\bigotimes_{i \in I} X_{i}$, and for $\left(l_{i}\right)_{i \in I} \in Y$, we have written $M_{i j l}(k, q) \equiv M_{j l_{l}}(k, q), C_{i l}(k, q) \equiv C_{l_{i}}(k, q)$. Every $Z_{l}$ is a nominator function which is admissible with respect to the degree set $\mathscr{E}$, consisting of all $\delta(H, \mathscr{S})=\overline{\operatorname{degr}}_{v \mid z} P$, $H \in \mathscr{H}^{\mathrm{UV}}((v)$ being the parametrization of $H$ and $(z)$ are the complementary parameters) and of all $\rho(H, \mathscr{S})=\operatorname{degr}_{u \mid w} P, H \in \mathscr{H}^{\mathbf{I R}}$ (parametrized by $(u)$ with complementary parameters $(w))$. Note that all $\delta(H, \mathscr{S})$ and $\rho(H, \mathscr{S})$ are independent of $\mathscr{S} \subseteq \mathscr{S}_{0}$.

Using (B-8), we get

$$
\overline{\mathscr{J}}_{\lambda}(q, \mu) \leqq \sum_{l \in Y} \int^{\lambda, \mathscr{L}} d^{4} k_{1} \cdots d^{4} k_{m} \frac{Z_{l}(k, q)}{E(k, q, \mu)} .
$$

The divergence degrees (4-17) and (4-20) are given by (4-25) and (4-26), respectively. Thus, all the conditions are met for the auxiliary theorem to apply to every integral on the right-hand side of (B-10). This proves the corollary.

\section{Appendix C. Proof of the Numerator Bounds}

In this appendix, Theorem 3 of Sect. 5 is proved. The proof is similar to that of the corresponding statement of [1], and below iwo lemmas are taken over literally. 
However, we have to consider IR- and UV-degrees simultaneously. Consequently, the proof is more tedious here.

For $\delta=\left(\delta_{1}, \ldots, \delta_{n}\right) \in \mathbf{N}_{0}^{n}, d \in \mathbf{N}, d \leqq n-1$ and multi-indices $b_{i}$ for $i=1, \ldots, n$, let

$$
g_{\delta \mid d}\left(b_{d+1}, \ldots, b_{n}\right)= \begin{cases}1 & \text { if }\left|b_{i}\right|+\cdots+\left|b_{n}\right|=\delta_{i}, \quad i=d+1, \ldots, n \\ 0 & \text { otherwise }\end{cases}
$$

and

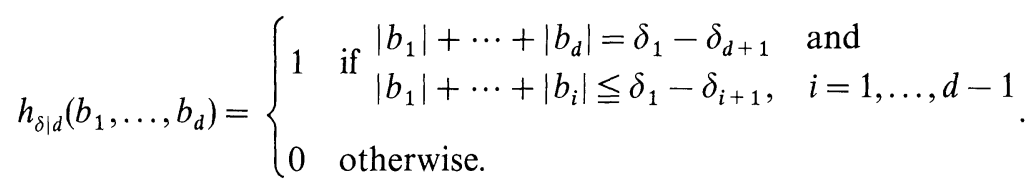

We state the preliminary

Lemma C.1. Let $F \in C^{\infty}$ be of the form $F\left(x_{1}, \ldots, x_{n}\right), x_{i} \in \mathbf{R}^{m_{i}}$, and $\delta_{i} \in \mathbf{N}_{0}=\{0,1,2, \ldots\}$ such that $\delta_{i} \geqq \delta_{k}$ if $i<k$, for all $i, k=1, \ldots, n$. Suppose

$$
F\left(x_{1}, \ldots, x_{j-1}, \lambda x_{j}, \ldots, \lambda x_{n}\right)=O\left(\lambda^{\delta_{j}}\right), \quad \lambda \rightarrow 0 ; \quad j=1, \ldots, n .
$$

Let $d \in \mathbf{N}, d \leqq n-1$. Then there exist $C^{\infty}$-functions $F_{b_{1} \cdots b_{n}}$, so that

$$
\begin{aligned}
& F\left(x_{1}, \ldots, x_{n}\right) \\
& \quad=\sum_{b_{1}, \ldots, b_{n}} h_{\delta \mid d}\left(b_{1}, \ldots, b_{d}\right) g_{\delta \mid d}\left(b_{d+1}, \ldots, b_{n}\right) x_{1}^{b_{1}} \cdots x_{d-1}^{b_{d-1}} y_{d}^{b_{d}} y_{d+1}^{b_{d+1}} \cdots y_{n}^{b_{n}} F_{b_{1} \cdots b_{n}}\left(x_{1}, \ldots, x_{n}\right),
\end{aligned}
$$

where

$$
\begin{aligned}
\left(y_{n}\right) & =\left(x_{n}\right) \\
\left(y_{n-1}\right) & =\left(x_{n-1}, x_{n}\right) \\
\vdots & \\
\left(y_{d}\right) & =\left(x_{d}, \ldots, x_{n}\right) .
\end{aligned}
$$

Proof. By successive application of Lemma 6.1 of [1] to $F$, we get

$$
F\left(x_{1}, \ldots, x_{n}\right)=\sum_{b_{d+1} \cdots b_{n}} g_{\delta \mid d}\left(b_{d+1}, \ldots, b_{n}\right) y_{d+1}^{b_{d+1}} \cdots y_{n}^{b_{n}} F_{b_{d+1} \cdots b_{n}}\left(x_{1}, \ldots, x_{n}\right),
$$

where $F_{b_{d+1} \cdots b_{n}} \in C^{\infty}$ and

$$
F_{b_{d+1} \cdots b_{n}}\left(x_{1}, \ldots, x_{j-1}, \lambda x_{j}, \ldots, \lambda x_{n}\right)=O\left(\lambda^{\delta^{-}-\delta_{d+1}}\right), \quad \lambda \rightarrow 0 ; \quad 1 \leqq j \leqq d .
$$

Applying Lemma 6.2 of [1] to $F_{b_{d+1} \cdots b_{n}}$ yields

$$
F_{b_{d+1} \cdots b_{n}}\left(x_{1}, \ldots, x_{n}\right)=\sum_{b_{1} \cdots b_{d}} h_{\delta \mid d}\left(b_{1}, \ldots, b_{d}\right) x_{1}^{b_{1}} \ldots x_{d-1}^{b_{d}-1} y_{d}^{b_{d}} F_{b_{1} \cdots b_{d} b_{d+1} \cdots b_{n}}\left(x_{1}, \ldots, x_{n}\right),
$$

where $F_{b_{1} \cdots b_{n}} \in C^{\infty}$. Inserting this into (C-3), the assertion follows.

From Lemma C.1, we derive a bound on a function $V \in \mathscr{C}_{m_{0}}^{c}$ if ordered sequences of subspaces in $\mathscr{H}^{\mathrm{IR}}$ and $\mathscr{H}^{\mathrm{UV}}$ with respect to a natural set $\mathscr{L}$ of line momenta are given. 
Lemma C.2. Let $\mathscr{S} \subseteq \mathscr{L}$ and

$$
u^{(1)}, \ldots, u^{(r)}, \quad v^{(1)}, \ldots, v^{(m-r)}
$$

be a basis of $\mathscr{L}$ with respect to $k$ such that $u^{(1)}, \ldots, u^{(r)}$ is a basis of $\mathscr{S}$ with respect to $k$. Let $H_{1}, \ldots, H_{t}$ be a sequence of subspaces of $\mathscr{H}^{\mathrm{IR}}$ which is ordered in $u$ with respect to the basis $(C-5)$. Denote the parameters of $H_{i} b y\left(z_{i}\right)$ and the complementary parameters by $\left(\underline{z}_{i}\right)$. Furthermore, let $K_{1}, \ldots, K_{s}$ be a sequence of classes of affine subspaces of $\mathscr{H}^{\mathrm{UV}}$ which is ordered in $v$ with respect to $(C-5)$. Denote the parameters of $K_{i}$ by $\left(w_{i}\right)$ and the complementary parameters by $\left(\underline{w}_{i}\right)$.

Consider a function $V(k, q ; a) \in \mathscr{C}_{m_{0}}^{c}$ for some $m_{0} \in \mathbf{Z}$ and assume that $(k a, q a)$ are bounded. Then $V$ admits an estimate of the form

$$
|V(k, q ; a)-P(k, q)| \leqq a^{p} \sum_{b_{1} \cdots b_{t}} f\left(b_{1}, \ldots, b_{t}\right)\left|z_{1}^{b_{1}} \cdots z_{t}^{b_{t}}\right| \cdot \sum_{l \in X}\left|Q_{l b_{1} \cdots b_{t}}(k, q)\right|,
$$

where

$$
f\left(b_{1}, \ldots, b_{t}\right)= \begin{cases}1 & \text { if }\left|b_{1}\right|+\cdots+\left|b_{i}\right|=\underline{\operatorname{degr}}_{\hat{s}_{i} \mid \underline{z}_{l}} V, \text { for all } i=1, \ldots, t \\ 0 & \text { otherwise. }\end{cases}
$$

$X$ is a finite set and $p \in \mathbf{N}$ is independent of the sequences and the basis. $Q_{l b_{1} \cdots b_{t}}$ are homogeneous polynomials, and $P(k, q)=\lim _{a \rightarrow 0} V(k, q ; a)$, satisfying

and

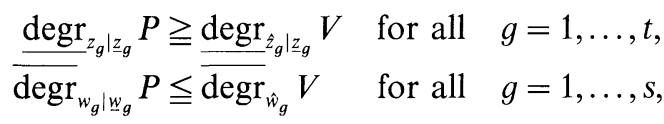

$$
\overline{\operatorname{degr}}_{w_{g} \mid \underline{w}_{g}} Q_{l b_{1} \cdot b_{t}} \leqq \overline{\operatorname{degr}}_{\hat{w}_{g}} V+p, \quad \text { for all } g=1, \ldots, s .
$$

Note that for $f\left(b_{1}, \ldots, b_{t}\right) \neq 0$

$$
\underline{\operatorname{degr}}_{z_{g} \mid \underline{z}_{g}} f\left(b_{1}, \ldots, b_{t}\right) z_{1}^{b_{1}} \cdots z_{t}^{b_{t}}=\underline{\operatorname{degr}}_{\hat{z}_{g} \mid \underline{z}_{g}} V, \quad g=1, \ldots, t .
$$

The polynomials $f\left(b_{1}, \ldots, b_{t}\right) z_{1}^{b_{1}} \cdots z_{t}^{b_{t}}$ depend only on the basis of $\mathscr{S}$, i.e., they are the same for all bases $(\mathrm{C}-5)$ with the same collection $u^{(1)}, \ldots, u^{(r)}$ and arbitrary $v^{(1)}, \ldots, v^{(m-r)}$. The integer $p$ can be chosen to be 1 if $P(k, q) \neq 0$. If $P(k, q) \equiv 0, p$ is the largest natural number so that $\lim _{a \rightarrow 0} V(k, q ; a) / a^{p} \neq \equiv$ exists.

Proof. 1. Write $V(k, q ; a)=F(k a, q a) / a^{m_{0}}$ and $F^{\prime}(x)=F(k(u, v, \tau \cdot q), \tau \cdot q)$ for fixed $q$ and variable $\tau^{8}$. We define variables $(x)=\left(x_{1}, \ldots, x_{s+t+1}\right)$ as follows

$$
\begin{aligned}
\left(w_{1}\right) & =\left(x_{1}\right) \\
\left(w_{2}\right) & =\left(x_{1}, x_{2}\right) \\
\vdots & \\
\left(w_{s}\right) & =\left(x_{1}, \ldots, x_{s}\right) \\
(v, \tau) & =\left(x_{1}, \ldots, x_{s+1}\right) \\
\left(z_{t}\right) & =\left(x_{s+2}, \ldots, x_{s+t+1}\right) \\
\vdots & \\
\left(z_{2}\right) & =\left(x_{s+t}, x_{s+t+1}\right) \\
\left(z_{1}\right) & =\left(x_{s+t+1}\right) .
\end{aligned}
$$

8 We write $\tau$ instead of $a$ to avoid misunderstandings 
For $H_{j}, j=1, \ldots, t$, set $\left(z_{j}\right)=\left(x_{s+t+2-j}, \ldots, x_{s+t+1}\right)$ ("internal" momenta of $\left.H_{j}\right)$ and $\left(\underline{z}_{j}\right)=\left(x_{1}, \ldots, x_{s+t+1-j}\right)$ ("external" momenta), so that $\left(z_{j}, \underline{z}_{j}\right)=\left(x_{1}, \ldots, x_{s+t+1}\right)$. Define $r_{s+t+1-j}=\operatorname{degr}_{\hat{z}_{j} \mid z_{j}} V$ for all $j=1, \ldots, t$. Similarly, for $K_{j}, j=1, \ldots, s$, set $\left(w_{j}\right)=\left(x_{1}, \ldots, x_{j}\right)$ and $\left(\underline{w}_{j}\right)=\left(x_{j+1}, \ldots, x_{s+t+1}\right)$, so that $\left(w_{j}, \underline{w}_{j}\right)=\left(x_{1}, \ldots, x_{s+t+1}\right)$. Define $r_{j}=m_{0}-\overline{\operatorname{degr}}_{\hat{w}} V$ for all $j=1, \ldots, s$. Then, by definition of the IR- and UV-degrees, $r_{1} \geqq r_{2} \geqq \cdots \geqq r_{s+t}$, and

$$
F^{\prime}\left(x_{1}, \ldots, x_{j}, \lambda x_{j+1}, \ldots, \lambda x_{s+t+1}\right)=O\left(\lambda^{r_{J}}\right), \quad \lambda \rightarrow 0 ; \quad j=1, \ldots, s+t .
$$

2. As in [1], define for $\delta \in \mathbf{Z}$

$$
\begin{aligned}
& P_{\delta}^{\prime}\left(x_{1}, \ldots, x_{s+t+1}\right)=\lim _{\lambda \rightarrow 0} \frac{F^{\prime}\left(\lambda x_{1}, \ldots, \lambda x_{s+t+1}\right)}{\lambda^{\delta}}, \\
& G\left(x_{1}, \ldots, x_{s+t+1}\right)=F^{\prime}\left(x_{1}, \ldots, x_{s+t+1}\right)-P_{m_{0}}^{\prime}\left(x_{1}, \ldots, x_{s+t+1}\right) .
\end{aligned}
$$

Let $r_{0} \in \mathbf{N}_{0}$ be the largest integer such that $P_{r_{0}}^{\prime}\left(x_{1}, \ldots, x_{s+t+1}\right) \not \equiv 0$ exists. Then

$$
G\left(x_{1}, \ldots, x_{j}, \lambda x_{j+1}, \ldots, \lambda x_{s+t+1}\right)=O\left(\lambda^{\hat{r}_{j}}\right) ; \quad \lambda \rightarrow 0, \quad 0 \leqq j \leqq s+t,
$$

where $\hat{r}_{j}=r_{j}$ for all $j=1, \ldots, s+t$ and

$$
\hat{r}_{0}=\left\{\begin{array}{lll}
r_{0} & \text { if } & m_{0}<r_{0} \\
r_{0}+1 & \text { if } & m_{0}=r_{0},
\end{array}\right.
$$

and hence $\hat{r}_{0} \geqq \hat{r}_{1} \geqq \cdots \geqq \hat{r}_{s+t}$. We now apply Lemma C.1 to $G$ with $d=s+1$, $n=s+t+1,\left(y_{i}\right)=\left(x_{i}, \ldots, x_{s+t+1}\right), i=s+1, \ldots, s+t+1$ :

$$
\begin{aligned}
& G\left(x_{1}, \ldots, x_{s+t+1}\right)=\sum_{b_{s+2} \cdots b_{s+t+1}} \tilde{f}\left(b_{s+2}, \ldots, b_{s+t+1}\right) y_{s+2}^{b_{s+2}} \cdots y_{s+t+1}^{b_{s+t+1}} \\
& \cdot \sum_{b_{1} \cdots b_{s+1}} \tilde{h}\left(b_{1}, \ldots, b_{s+1}\right) x_{1}^{b_{1}} \cdots x_{s}^{b_{s}} y_{s+1}^{b_{s+1}} F_{b_{1} \cdots b_{s+t+1}}\left(x_{1}, \ldots, x_{s+t+1}\right),
\end{aligned}
$$

where

$$
\begin{aligned}
& \tilde{f}\left(b_{s+2}, \ldots, b_{s+t+1}\right)= \begin{cases}1 & \text { if }\left|b_{i}\right|+\cdots+\left|b_{s+t+1}\right|=\hat{r}_{i-1}, \quad i=s+2, \ldots, s+t+1 \\
0 & \text { otherwise, }\end{cases} \\
& \tilde{h}\left(b_{1}, \ldots, b_{s+1}\right)= \begin{cases}1 & \text { if } \begin{array}{l}
\left|b_{1}\right|+\cdots+\left|b_{s+1}\right|=\hat{r}_{0}-\hat{r}_{s+1} \text { and } \\
\left|b_{1}\right|+\cdots+\left|b_{i}\right| \leqq \hat{r}_{0}-\hat{r}_{i} \text { for all } i=1, \ldots, s
\end{array} \\
0 & \text { otherwise }\end{cases}
\end{aligned}
$$

and $F_{b_{1} \cdots b_{s+t+1}} \in C^{\infty}$. For bounded $(k a, q a)$ and $\tau=1$, using $\left(y_{i}\right)=\left(z_{s+t+2-i}\right)$ for $i=s+2, \ldots, s+t+1$, we get

$$
\left|G\left(x_{1} a, \ldots, x_{s+t+1} a\right)\right|_{\tau=1} \leqq a^{\hat{r}_{0}} \sum_{b_{1} \cdots b_{t}} f\left(b_{1}, \ldots, b_{t}\right)\left|z_{1}^{b_{1}} \cdots z_{t}^{b_{t}}\right| \sum_{l \in X}\left|Q_{l b_{1} \cdot b_{t}}(k, q)\right|,
$$

where $f\left(b_{1}, \ldots, b_{t}\right)$ is defined in (C-9) and $X$ is a finite set. $Q_{l b_{1} \cdots b_{t}}$ are polynomials, satisfying

$$
\overline{\operatorname{degr}}_{w_{g} \mid \underline{w}_{g}} Q_{l b_{1} \cdots b_{t}} \leqq \hat{r}_{0}-\hat{r}_{g}=\left(\hat{r}_{0}-m_{0}\right)+\overline{\operatorname{degr}}_{\hat{w}_{g}} V, \quad g=1, \ldots, s .
$$


Finally, note that

$$
\left.P(k, q) \equiv \lim _{a \rightarrow 0} \frac{1}{a^{m_{0}}} V(k, q ; a) \equiv P_{m_{0}}^{\prime}\left(x_{1}, \ldots, x_{s+t+1}\right)\right|_{\tau=1} .
$$

Then

$$
\begin{array}{ll}
\underline{\operatorname{degr}}_{z_{g} \mid \underline{z}_{g}} P \geqq \hat{r}_{s+t+1-g}=\underline{\operatorname{degr}}_{\hat{g}_{g} \mid \underline{z}_{g}} V, \quad g=1, \ldots, t, \\
\overline{\operatorname{degr}}_{w_{g} \mid \underline{w}_{g}} P \leqq m_{0}-\hat{r}_{g}=\overline{\operatorname{degr}}_{\hat{w}_{g}} V, \quad g=1, \ldots, s,
\end{array}
$$

and

$$
\begin{aligned}
|V(k, q ; a)-P(k, q)| & =\frac{1}{a^{m_{0}}}\left|G\left(x_{1} a, \ldots, x_{s+t+1} a\right)\right|_{\tau=1} \\
& \leqq a^{p} \sum_{b_{1} \cdots b_{t}} f\left(b_{1}, \ldots, b_{t}\right)\left|z_{1}^{b_{1}} \cdots z_{t}^{b_{t}}\right| \sum_{l \in X}\left|Q_{l b_{1} \cdots b_{t}}(k, q)\right|,
\end{aligned}
$$

where $p=\hat{r}_{0}-m_{0} \in \mathbf{N}$.

Proof of Theorem 3. Using Lemma C.2, the proof of straightforward. We have to show the validity of an estimate

$$
|V(k, q ; a)-P(k, q)| \leqq a^{p} \sum_{b \in B} Z_{b}(k, q),
$$

where every $a^{p} Z_{b}(k, q)$ is a nominator function which is admissible with respect to the degree set $\mathscr{D}$, defined at the beginning of Sect. 5 .

At first, let $\mathscr{S} \subseteq \mathscr{M}$ (cp. (3-4)) be a given subset and

$$
u^{(1)}, \ldots, u^{(r)}, \quad v^{(1)}, \ldots, v^{(m-r)}
$$

a basis of $\mathscr{L}$ with respect to $k$ so that $u^{(1)}, \ldots, u^{(r)}$ is a basis of $\mathscr{S}$ with respect to $k$. Let $H_{1}, \ldots, H_{t} \in \mathscr{H}^{\mathrm{IR}}$ be a sequence which is ordered in $u$ with respect to the basis (C-17), and $K_{1}, \ldots, K_{s} \in \mathscr{H}^{\mathrm{UV}}$ a sequence which is ordered in $v$ with respect to (C-17). Using Lemma C.2, $V \in \mathscr{C}_{m_{0}}^{c}$ can be estimated by

$$
|V(k, q ; a)-P(k, q)| \leqq a^{p} \sum_{j \in J}\left|M_{j}(u)\right| \sum_{l \in X}\left|Q_{j l}(k, q)\right|,
$$

where $P(k, q)=\lim _{a \rightarrow 0} V(k, q ; a), p \in \mathbf{N}$ is determined by the function $V$, and $J, X$ are finite sets. $M_{j}$ and $Q_{j l}$ are homogeneous polynomials satisfying

$$
\underline{\operatorname{degr}}_{z_{g} \mid \underline{z}_{g}} M_{j} \geqq \underline{\operatorname{degr}}_{\underline{t}_{g} \mid \underline{z}_{g}} V \text { for all } g=1, \ldots, t \text { and for all } j \in J,
$$

where $\left(z_{g}\right)$ are the parameters of $H_{g}$ and $\left(z_{g}, \underline{z}_{g}\right)=(u, v)$, and

$$
\overline{\operatorname{degr}}_{w_{g} \mid \underline{w}_{g}} Q_{j l} \leqq \overline{\operatorname{degr}}_{\hat{w}_{g}}+p \text { for all } g=1, \ldots, s \text { and for all } j \in J, l \in X,
$$

$\left(w_{g}\right)$ being the parameters of $K_{g}$ and $\left(w_{g}, \underline{w}_{g}\right)=(u, v)$.

We now make an estimate of the form (C-18)

a. For all sequences of subspaces of $\mathscr{H}^{\mathrm{UV}}$ which are ordered in $v$.

b. For all bases $(\mathrm{C}-17)$ of $\mathscr{L}$ with fixed $u^{(1)}, \ldots, u^{(r)}$, i.e. for given $(u)$ we consider 
all possible choices of $(v)$ such that (C-17) is a basis of $\mathscr{L}$. Note that by such changes of the basis the IR-degrees $\operatorname{degr}_{\hat{}_{g} \mid \underline{z}_{g}} V$ do not change'.

We get

$$
|V(k, q ; a)-P(k, q)| \leqq a^{p} \sum_{l \in Y}\left|M_{l}(u)\right| \min _{i \in \mathbf{K}}\left|\tilde{Q}_{i l}(k, q)\right|,
$$

where $Y, K$ are finite sets. For every basis $(u, v)$ of $\mathscr{L}$ with given $(u)$ and every sequence $K_{1}, \ldots, K_{s} \in \mathscr{H}^{\mathrm{UV}}$ which is ordered in $v$ with respect to this basis, there is an $i \in K$, so that the polynomials $\tilde{Q}_{i l}$ satisfy

$$
\overline{\operatorname{degr}}_{w_{g} \mid \underline{w}_{g}} \tilde{Q}_{i l} \leqq \overline{\operatorname{degr}}_{\hat{w}_{g}} V+p \text { for all } g=1, \ldots, s \text { and for all } l \in Y .
$$

$\left(w_{g}\right)$ are the parameters of $K_{g}$ and $\left(\underline{w}_{g}\right)$ the complementary parameters.

Next, we consider

a. All sequences of subspaces of $\mathscr{H}^{\mathrm{IR}}$ which are ordered in $u$,

b. All bases (C-17) such that $u^{(1)}, \ldots, u^{(r)}$ is an arbitrary basis of $\mathscr{S}$. By the corresponding changes of a basis, the UV-degrees $\overline{\operatorname{degr}}_{\hat{w}_{g}} V$ do not change ${ }^{10}$.

We get

$$
|V(k, q ; a)-P(k, q)| \leqq a^{p} \sum_{b \in B} \min _{j \in J}\left|M_{j b}(u, q)\right| \cdot \min _{l \in K_{b}}\left|C_{l b}(k, q)\right|,
$$

where $B, J, K_{b}$ are finite sets and $M_{j b}, C_{l b}$ are polynomials. For every basis (C-17) of $\mathscr{L}$ such that $u^{(1)}, \ldots, u^{(r)}$ is a basis of $\mathscr{S}$, the polynomials $M_{j b}$ depend only on $u$ and the external momenta $q$. Furthermore, for every sequence $H_{1}, \ldots, H_{t} \in \mathscr{H}^{\mathrm{IR}}$ which is ordered in $u$ with respect to (C-17) there exists $j \in J$, so that

$$
\underline{\operatorname{degr}}_{z_{g} \mid \underline{z}_{g}} M_{j b} \geqq \underline{\operatorname{degr}}_{\underline{q}_{g} \mid \underline{z}_{g}} V \text { for all } g=1, \ldots, t \text { and for all } b \in B,
$$

where $\left(z_{g}\right)$ are the parameters of $H_{g}$ and $\left(z_{g}, \underline{z}_{g}\right)=(u, v)$. For every sequence $K_{1}, \ldots, K_{s} \in \mathscr{H}^{\mathrm{UV}}$ which is ordered in $v$ with respect to (C-17) and for every $b \in B$ there is $l \in K_{b}$, so that

$$
\overline{\operatorname{degr}}_{w_{g} \mid \underline{w}_{g}} C_{l b} \leqq \overline{\operatorname{degr}}_{\hat{w}_{g}} V+p \text { for all } g=1, \ldots, s .
$$

${ }^{9}$ A change of basis

is given by

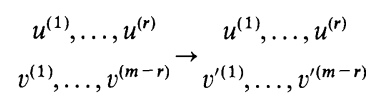

where $B$ is an invertible matrix.

$$
\begin{aligned}
& u^{\prime}=u \\
& v^{\prime}=A u+B v+C q,
\end{aligned}
$$

10 We have

$$
\begin{aligned}
u^{\prime} & =D u+E q \\
v^{\prime} & =v,
\end{aligned}
$$

where $D$ is invertible. The change of the basis of $\mathscr{S}$ depends on the external momenta $q$, hence the polynomials $M_{l}$ are dependent on $u$ and $q$ 
$\left(w_{g}\right)$ are the parameters of $K_{g}$ and $\left(w_{g}, \underline{w}_{g}\right)=(u, v)$. This means that, taking on the right-hand side of $(\mathrm{C}-19)$ the minimum over all $\mathscr{S} \subseteq \mathscr{M}$, we get an estimate of the form $(\mathrm{C}-16)$, where all functions $a^{p} Z_{b}(k, q)$ are nominator functions which are admissible with respect to the degree set $\mathscr{D}$. This proves Theorem 3.

Acknowledgement. The author wishes to thank M. Lüscher for many discussions.

\section{References}

1. Reisz, T.: A power counting theorem for Feynman integrals on the lattice. Commun. Math. Phys. 116, (1988)

2. Lowenstein, J. H., Zimmermann, W.: The power counting theorem for Feynman integrals with massless propagators. Commun. Math. Phys. 44, 73-86 (1975)

3. Bandelloni, G., Becchi, C., Blasi, A., Collina, R.: Renormalization of models with radiative mass generation. Commun. Math. Phys. 67, 147-178 (1978)

4. Lowenstein, J. H., Zimmermann, W.: On the formulation of theories with zero-mass propagators. Nucl. Phys. B86, 77 (1975)

5. Lowenstein, J. H.: Convergence theorems for renormalized Feynman integrals with zero-mass propagators. Commun. Math. Phys. 47, 53-68 (1976)

6. Reisz, T.: Renormalization of lattice Feynman integrals with massless propagators. Commun. Math. Phys. (in press)

Communicated by A. Jaffe

Received October 15, 1987 